\title{
Critical points of multidimensional random Fourier series: Central limits
}

\author{
LIVIU I. NICOLAESCU ${ }^{1}$ \\ ${ }^{1}$ Department of Mathematics, University of Notre Dame, Notre Dame, IN 46556-4618, USA. \\ E-mail: Inicolae@nd.edu; url: http://www3.nd.edu/ Inicolae/
}

We investigate certain families $X^{\hbar}, 0<\hbar \ll 1$ of stationary Gaussian random smooth functions on the $m$-dimensional torus $\mathbb{T}^{m}:=\mathbb{R}^{m} / \mathbb{Z}^{m}$ approaching the white noise as $\hbar \rightarrow 0$. We show that there exists universal constants $c_{1}, c_{2}>0$ such that for any cube $B \subset \mathbb{R}^{m}$ of size $r \leq 1 / 2$, the number of critical points of $X^{\hbar}$ in the region $B \bmod \mathbb{Z}^{m} \subset \mathbb{T}^{m}$ has mean $\sim c_{1} \operatorname{vol}(B) \hbar^{-m}$, variance $\sim c_{2} \operatorname{vol}(B) \hbar^{-m}$, and satisfies a central limit theorem as $\hbar \searrow 0$.

Keywords: central limit theorem; critical points; Gaussian Hilbert spaces; Gaussian random functions; Kac-Rice formula; Wiener chaos

\section{The main results}

\subsection{By way of motivation}

During the final years of his life, V.I. Arnold [4-7] investigated the Morse theoretic properties of trigonometric polynomials on higher dimensional tori. We describe below one direction of his investigation.

Fix a convex polygon $\mathcal{P}$ in $\mathbb{R}^{m}$, with vertices in the lattice $\mathbb{Z}^{m}$. We assume that $\mathcal{P}$ is admissible, that is, it is symmetric with respect to the origin and it is invariant with respect to the natural action of the symmetric group $S_{m}$ on $\mathbb{R}^{m}$.

Consider the vector space $\mathbf{V}_{\mathcal{P}}$ of trigonometric polynomials on $\mathbb{T}^{m}=\mathbb{R}^{m} / \mathbb{Z}^{m}$ whose Newton's polyhedra are contained in $\mathcal{P}$, i.e., polynomials of the form

$$
f(\boldsymbol{\theta})=\sum_{\mathbf{k} \in \mathcal{P} \cap \mathbb{Z}^{m}}\left(A_{\mathbf{k}} \cos (2 \pi\langle\mathbf{k}, \boldsymbol{\theta}\rangle)+B_{\mathbf{k}} \sin (2 \pi\langle\mathbf{k}, \boldsymbol{\theta}\rangle)\right), \quad \boldsymbol{\theta} \in \mathbb{T}^{m} .
$$

We can think of $f$ as a random polynomial by declaring the coefficients $A_{\mathbf{k}}$ and $B_{\mathbf{k}}$ to be independent standard normal random variables. We denote by $\mathbf{N}^{\mathcal{P}}(f)$ the number of critical points of $f(\theta)$. We denote $\mathbf{N}_{\max }^{\mathcal{P}}$ the (essential) supremum of the positive random variable $\mathbf{N}^{\mathcal{P}}(f)$. The results of Bernshtein [12] and Kouchnirenko [23] imply the very rough upper bound

$$
\mathbf{N}_{\max }^{\mathcal{P}} \leq K_{m}(\mathcal{P}):=m ! \operatorname{vol}(\mathcal{P}) .
$$

In dimension $m=2$, Arnold proved that for several classes of admissible polyhedra $\mathcal{P}$ the upper bound $K_{m}(\mathcal{P})$ is very close to being optimal. He achieved this by producing nontrivial lower bounds for $\mathbf{N}_{\max }^{\mathcal{P}}$ using techniques from real algebraic geometry. 
There exists an obvious probabilistic lower bound for $\mathbf{N}_{\max }^{\mathcal{P}}$, namely

$$
\mathbf{N}_{\max }^{\mathcal{P}} \geq Z^{\mathcal{P}}:=\mathbb{E}\left[\mathbf{N}^{\mathcal{P}}(f)\right]
$$

The Kac-Rice formula shows that for the polyhedra considered by Arnold the lower bound $Z^{\mathcal{P}}$ is very close to the upper bound $K_{m}(\mathcal{P})$. Moreover, for any admissible polyhedron $\mathcal{P}$ there exists an explicit constant $C=C_{m}(\mathcal{P})>0$ such that

$$
Z^{\nu \mathcal{P}} \sim C_{m}(\mathcal{P}) \nu^{m} \quad \text { as } v \rightarrow \infty \text { inside } \mathbb{N} .
$$

Clearly $K_{m}(\nu \mathcal{P})=K_{m}(\mathcal{P}) \nu^{m}$ so the mean $Z^{\nu \mathcal{P}}$ and the Bernshtein-Kouchnirenko very rough upper bound $K_{m}(\nu \mathcal{P})$ have the same rate of growth as $v \rightarrow \infty$. These observations suggest that the random variables $\mathbf{N}^{\nu \mathcal{P}}(f)$ might concentrate near their means as $\nu \rightarrow \infty$.

We can reformulate the above facts as follows. Denote by $w$ the indicator function of $\mathcal{P}$. We can describe the random trigonometric polynomial (1.1) in the form

$$
f(\boldsymbol{\theta})=\sum_{\mathbf{k} \in \mathbb{Z}^{m}} w(\mathbf{k})\left(A_{\mathbf{k}} \cos (2 \pi\langle\mathbf{k}, \boldsymbol{\theta}\rangle)+B_{\mathbf{k}} \sin (2 \pi\langle\mathbf{k}, \boldsymbol{\theta}\rangle)\right) .
$$

The rescaling $\mathcal{P} \mapsto \nu \mathcal{P}$ corresponds to a new random polynomial

$$
f^{\hbar}(\boldsymbol{\theta})=\sum_{\mathbf{k} \in \mathbb{Z}^{m}} w(\hbar \mathbf{k})\left(A_{\mathbf{k}} \cos (2 \pi\langle\mathbf{k}, \boldsymbol{\theta}\rangle)+B_{\mathbf{k}} \sin (2 \pi\langle\mathbf{k}, \boldsymbol{\theta}\rangle)\right), \quad \hbar=v^{-1}
$$

In this paper, we investigate random Fourier series of the form (1.4), where $w$ is a nonnegative, radially symmetric, Schwartz function. The main results in this paper will show that as $\hbar \rightarrow 0$ the number of critical points of $f^{\hbar}$ in a fixed open set of $\mathbb{T}^{m}$ satisfies a central limit theorem.

The relationship with Bernstein-Koushnirenko inequality should be clear. Suppose that $w(\lambda)>0$ for $|\lambda|<1$ and $w(\lambda)=0$ for $|\lambda|>0$. For $\hbar>0$ denote by $\mathcal{P}_{\hbar}$ the convex hull of the lattice points in the ball of radius $\hbar^{-1}$ centered at the origin. Our results show that

$$
\log \mathbf{N}_{\max }^{\mathcal{P}_{\hbar}} \sim-m \log \hbar, \quad \hbar \rightarrow 0,
$$

and the number of critical points of $f^{\hbar}$ is highly concentrated near its mean that has the form const $\times \hbar^{-m}$. The next subsections contain the precise statements.

\subsection{The main problem}

For any $\hbar>0$, we denote by $\mathbb{T}_{\hbar}^{m}$ the $m$-dimensional torus $\mathbb{R}^{m} / \mathbb{Z}^{m}$ with angular coordinates $\theta_{1}, \ldots, \theta_{m} \in \mathbb{R} / \mathbb{Z}$ equipped with the flat metric

$$
g_{\hbar}:=\sum_{j=1}^{m} \hbar^{-2}\left(d \theta_{j}\right)^{2}
$$


For a measurable subset $S \subset \mathbb{T}^{m}$ we denote by $\operatorname{vol}_{\hbar}(S)$ its volume with respect to the metric $g_{\hbar}$, and we set vol $:=\left.\operatorname{vol}_{\hbar}\right|_{\hbar=1}$. Hence,

$$
\operatorname{vol}_{\hbar}\left(\mathbb{T}^{m}\right)=\hbar^{-m} \operatorname{vol}\left(\mathbb{T}^{m}\right)=\hbar^{-m}
$$

The eigenvalues of the corresponding Laplacian $\Delta_{\hbar}=-\hbar^{2} \sum_{k=1}^{m} \partial_{\theta_{k}}^{2}$ are

$$
\lambda^{\hbar}(\mathbf{k})=\hbar^{2} \lambda(\mathbf{k}), \quad \lambda(\mathbf{k}):=|2 \pi \mathbf{k}|^{2}, \quad \mathbf{k}=\left(k_{1}, \ldots, k_{m}\right) \in \mathbb{Z}^{m}
$$

Denote by $\prec$ the lexicographic order on $\mathbb{R}^{m}$. An orthonormal basis of $L^{2}\left(\mathbb{T}_{\hbar}^{m}\right)$ is given by the functions $\left(\psi_{\mathbf{k}}^{\hbar}\right)_{\mathbf{k} \in \mathbb{Z}^{m}}$, where

$$
\psi_{\mathbf{k}}^{\hbar}(\boldsymbol{\theta})=\hbar^{\frac{m}{2}} \psi_{\mathbf{k}}(\boldsymbol{\theta}), \quad \psi_{\mathbf{k}}(\boldsymbol{\theta}):= \begin{cases}1, & \mathbf{k}=\overrightarrow{0} 0 \\ \sqrt{2} \sin 2 \pi\langle\mathbf{k}, \boldsymbol{\theta}\rangle, & \mathbf{k} \succ \overrightarrow{0} \\ \sqrt{2} \cos 2 \pi\langle\mathbf{k}, \boldsymbol{\theta}\rangle, & \mathbf{k} \prec \overrightarrow{0} .\end{cases}
$$

Fix a nonnegative, even Schwarz function $w \in \mathcal{S}(\mathbb{R})$, set $w_{\hbar}(t):=w(\hbar t)$ so that

$$
w\left(\sqrt{\lambda^{\hbar}(\mathbf{k})}\right)=w_{\hbar}(\sqrt{\lambda(\mathbf{k})})
$$

Consider the random function given by the random Fourier series

$$
\begin{aligned}
X^{\hbar}(\boldsymbol{\theta}) & =\sum_{\mathbf{k} \in \mathbb{Z}^{m}} w\left(\sqrt{\lambda^{\hbar}(\mathbf{k})}\right)^{\frac{1}{2}} N_{\mathbf{k}} \psi_{\mathbf{k}}^{\hbar}(\boldsymbol{\theta})=\hbar^{\frac{m}{2}} \sum_{\mathbf{k} \in \mathbb{Z}^{m}} w_{\hbar}(\sqrt{\lambda(\mathbf{k})})^{\frac{1}{2}} N_{\mathbf{k}} \psi_{\mathbf{k}}(\boldsymbol{\theta}) \\
& =\hbar^{\frac{m}{2}} \sum_{\mathbf{k} \in \mathbb{Z}^{m}} w(2 \pi \hbar|\mathbf{k}|)^{\frac{1}{2}}\left(A_{\mathbf{k}} \cos (2 \pi\langle\mathbf{k}, \boldsymbol{\theta}\rangle)+B_{\mathbf{k}} \sin (2 \pi\langle\mathbf{k}, \boldsymbol{\theta}\rangle)\right),
\end{aligned}
$$

where the coefficients $A_{\mathbf{k}}, B_{\mathbf{k}}, N_{\mathbf{k}}, \mathbf{k} \in \mathbb{Z}^{m}$, are independent standard normal random variables such that

$$
A_{\mathbf{k}}+A_{-\mathbf{k}}=\sqrt{2} N_{\mathbf{k}}, \quad B_{\mathbf{k}}+B_{-\mathbf{k}}=\sqrt{2} N_{-\mathbf{k}}, \quad \forall \mathbf{k} \succ 0 .
$$

Note that if $w \equiv 1$ in a neighborhood of 0 , then the random function $\hbar^{-m / 2} X^{\hbar}$ converges to a Gaussian white-noise on $\mathbb{T}^{m}$ and, extrapolating, we can think of the $\hbar \rightarrow 0$ limits in this paper as white-noise limits.

The random function $X^{\hbar}(\boldsymbol{\theta})$ is a.s. smooth and Morse. For any Borel set $\mathcal{B} \subset \mathbb{T}^{m}$ we denote by $\mathbf{Z}\left(X^{\hbar}, \mathcal{B}\right)$ the number of critical points of $X^{\hbar}$ in $\mathcal{B}$. In [31], (see also Theorem 1.3(i) + (ii) in this paper) we have shown that there exist constants $C=C_{m}(w)>0, S=S_{m}(w) \geq 0$ such that, for any open set $\mathcal{O} \subset \mathbb{T}^{m}$,

$$
\begin{aligned}
\mathbb{E}\left[\mathbf{Z}\left(X^{\hbar}, \mathcal{O}\right)\right] & \sim C_{m}(w) \hbar^{-m} \operatorname{vol}(\mathcal{O}) & \text { as } \hbar \rightarrow 0, \\
\operatorname{var}\left[\mathbf{Z}\left(X^{\hbar}, \mathcal{O}\right)\right] & \sim S_{m}(w) \hbar^{-m} \operatorname{vol}(\mathcal{O}) & \text { as } \hbar \rightarrow 0
\end{aligned}
$$


Note that (1.6a) states that as $\hbar \rightarrow 0$ we should expect that the critical points of $X^{\hbar}$ will distribute uniformly with density $C_{m}(w) \hbar^{-m}$, while (1.6b) shows that this limiting equidistribution happens with high probability. In [31], we described the constants $C_{m}(w)$ and $S_{m}(w)$ explicitly as certain rather complicated Gaussian integrals, and we conjectured that $S_{m}(w)$ is actually strictly positive.

In this paper, we describe the manner in which the random variable $\mathbf{Z}\left(X^{\hbar}, \mathcal{O}\right)$ concentrates around its mean as $\hbar \rightarrow 0$. More precisely, we show that indeed $S_{m}(w)>0$, and we prove a central limit theorem stating that, as $\hbar \rightarrow 0$, the random variables

$$
\zeta_{\hbar}(\mathcal{O}):=\left(\frac{\hbar}{\operatorname{vol}(\mathcal{O})}\right)^{\frac{m}{2}}\left(\mathbf{Z}\left(X^{\hbar}, \mathcal{O}\right)-\mathbb{E}\left[\mathbf{Z}\left(X^{\hbar}, \mathcal{O}\right)\right]\right)
$$

converge in law to a nondegenerate normal random variable $\sim \mathcal{N}\left(0, S_{m}(w)\right)$.

We achieve this by relying on the central limit theorems of Nourdin and Peccatti $[33,34]$. This requires placing the problem within a Gaussian Hilbert space context.

\subsection{The Wiener chaos setup}

Let $\mathbf{x}=\left(x_{1}, \ldots, x_{m}\right)$ denote the standard Euclidean coordinates on $\mathbb{R}^{m}$. For $\mathbf{p}_{0} \in \mathbb{R}^{m}$ and $R>0$ we set

$$
\widehat{B}_{R}\left(\mathbf{p}_{0}\right):=\left\{\mathbf{x} \in \mathbb{R}^{m} ;\left|\mathbf{x}-\mathbf{p}_{0}\right|_{\infty} \leq \frac{R}{2}\right\}, \quad \widehat{B}_{R}=\widehat{B}_{R}(0)=\left[-\frac{R}{2}, \frac{R}{2}\right]^{m} .
$$

For $r \in(0,1]$ and denote by $B_{r}$ the image of the cube $\widehat{B}_{r}$ in the quotient $\mathbb{R}^{m} / \mathbb{T}^{m}$. Thus, for $r<1$, $B_{r}$ is a cube on the torus centered at 0 , while $B_{1}=\mathbb{T}^{m}$.

We identify the tangent space $T_{0} \mathbb{T}_{\hbar}^{m}$ with $\mathbb{R}^{m}$ and we denote by $\exp _{\hbar}$ the exponential map $\exp _{\hbar}: \mathbb{R}^{m} \rightarrow \mathbb{T}_{\hbar}^{m}$ defined by the metric $g_{\hbar}$. In the coordinates $\mathbf{x}$ on $\mathbb{R}^{m}$ and $\boldsymbol{\theta}$ on $\mathbb{T}^{m}$, this map is described by $\boldsymbol{\theta}=\hbar \mathbf{x} \bmod \mathbb{Z}^{m}$. Using this map, we obtain by pullback a $\left(\hbar^{-1} \mathbb{Z}\right)^{m}$-periodic random function on $\mathbb{R}^{m}$,

$$
Y^{\hbar}(\mathbf{x}):=\left(\exp _{\hbar}^{*} X^{\hbar}\right)(\mathbf{x})=\hbar^{\frac{m}{2}} \sum_{\mathbf{k} \in \mathbb{Z}^{m}} w(2 \pi \hbar|\mathbf{k}|)^{\frac{1}{2}}\left(A_{\mathbf{k}} \cos (2 \pi \hbar\langle\mathbf{k}, \mathbf{x}\rangle)+B_{\mathbf{k}} \sin (2 \pi \hbar\langle\mathbf{k}, \mathbf{x}\rangle)\right) .
$$

We denote by $\mathbf{Z}\left(Y^{\hbar}, \mathcal{B}\right)$ the number of critical points of $Y^{\hbar}$ in the Borel set $\mathcal{B} \subset \mathbb{R}^{m}$. Note that

$$
\mathbf{Z}\left(X^{\hbar}, B_{r}\right)=\mathbf{Z}\left(Y^{\hbar}, \widehat{B}_{\hbar^{-1} r}\right), \quad \forall r \in(0,1] .
$$

The main goal of this paper is to investigate the behavior of the random variable $\mathbf{Z}\left(X^{\hbar}, B_{r}\right)$ as $\hbar \searrow 0$.

A simple computation shows that the covariance kernel of $Y^{\hbar}$ is

$$
\mathcal{K}^{\hbar}(\mathbf{x}, \mathbf{y})=\hbar^{m} \sum_{\mathbf{k} \in \mathbb{Z}^{m}} w(2 \pi \hbar|\mathbf{k}|) \exp (-2 \pi \hbar \mathbf{i}\langle\mathbf{k}, \mathbf{z}\rangle), \quad \mathbf{z}=\mathbf{y}-\mathbf{x} .
$$


Define

$$
\phi_{\mathbf{z}}: \mathbb{R}^{m} \rightarrow \mathbb{C}, \quad \phi_{\mathbf{z}}(\xi):=e^{-\mathbf{i}\langle\xi, \mathbf{z}\rangle} w(|\xi|) .
$$

Using the Poisson formula [20], Section 7.2, we deduce that for any $a>0$, we have

$$
\sum_{\mathbf{k} \in \mathbb{Z}^{m}} \phi_{\mathbf{z}}\left(\frac{2 \pi}{a} \mathbf{k}\right)=\left(\frac{a}{2 \pi}\right)^{m} \sum_{\boldsymbol{v} \in \mathbb{Z}^{m}} \widehat{\phi}_{\mathbf{z}}(a \boldsymbol{v})
$$

where, for any $u=u(\xi) \in \mathcal{S}\left(\mathbb{R}^{m}\right)$, we denote by $\widehat{u}(\mathbf{t})$ its Fourier transform

$$
\widehat{u}(\mathbf{t}):=\int_{\mathbb{R}^{m}} e^{-\mathbf{i}\langle\boldsymbol{\xi}, \mathbf{t}\rangle} u(\boldsymbol{\xi})|d \xi| .
$$

If we let $a=\hbar^{-1}$, then we deduce

$$
\mathcal{K}^{\hbar}(\mathbf{x}, \mathbf{y})=\frac{1}{(2 \pi)^{m}} \sum_{\boldsymbol{v} \in \mathbb{Z}^{m}} \widehat{\phi_{\mathbf{z}}}\left(\hbar^{-1} \boldsymbol{v}\right) .
$$

Define $V: \mathbb{R}^{m} \rightarrow \mathbb{R}$ by

$$
V(\mathbf{t}):=\frac{1}{(2 \pi)^{m}} \widehat{w}(\mathbf{t})=\frac{1}{(2 \pi)^{m}} \int_{\mathbb{R}^{m}} e^{-\mathbf{i}\langle\boldsymbol{\xi}, \mathbf{t}\rangle} w(|\boldsymbol{\xi}|) d \boldsymbol{\xi} .
$$

Then

$$
\widehat{\phi_{\mathbf{z}}}(\mathbf{t})=V(\mathbf{z}+\mathbf{t})
$$

We deduce

$$
\mathcal{K}^{\hbar}(\mathbf{x}, \mathbf{y})=\sum_{\boldsymbol{v} \in \mathbb{Z}^{m}} V\left(\mathbf{z}+\frac{1}{\hbar} \boldsymbol{v}\right), \quad \mathbf{z}=\mathbf{y}-\mathbf{x}
$$

We set

$$
V^{\hbar}(\mathbf{z}):=\sum_{\boldsymbol{v} \in \mathbb{Z}^{m}} V\left(\mathbf{z}+\frac{1}{\hbar} \boldsymbol{v}\right) .
$$

The function $V^{\hbar}$ is $\left(\hbar^{-1} \mathbb{Z}\right)^{m}$-periodic and moreover,

$$
\mathcal{K}^{\hbar}(\mathbf{x}, \mathbf{y})=V^{\hbar}(\mathbf{z}), \quad \mathbf{z}:=\mathbf{y}-\mathbf{x} .
$$

The region $\widehat{B}_{1 / \hbar}=\left[-(2 \hbar)^{-1},(2 \hbar)^{-1}\right]^{m} \subset \mathbb{R}^{m}$ is a fundamental domain for the action of the lattice $\left(\hbar^{-1} \mathbb{Z}\right)^{m}$ on $\mathbb{R}^{m}$. From the special form (1.11) of $V^{\hbar}$ and the fact that $V$ is a Schwarz function we deduce that for any positive integers $k, N$ we have

$$
\left\|V^{\hbar}-V\right\|_{C^{k}\left(\widehat{B}_{1 / \hbar}\right)}=O\left(\hbar^{N}\right) \quad \text { as } \hbar \searrow 0
$$


where $\|-\|_{C^{k}\left(\widehat{B}_{1 / \hbar}\right)}$ denotes the $C^{k}$-norm

$$
\|f\|_{C^{k}\left(\widehat{B}_{1 / \hbar}\right)}=\sup _{\mathbf{x} \in\left(\widehat{B}_{1 / \hbar}\right),|\alpha| \leq k}\left|\partial_{\mathbf{x}}^{\alpha} f(x)\right| .
$$

From (1.8), we deduce that $Y^{\hbar}$ is a stationary Gaussian random function on $\mathbb{R}^{m}$ and its spectral measure is

$$
\mu^{\hbar}(d \boldsymbol{\xi})=\frac{1}{(2 \pi)^{m}} \sum_{\boldsymbol{v} \in(2 \pi \hbar \mathbb{Z})^{m}}(2 \pi \hbar)^{m} w(\boldsymbol{v}) \delta_{\boldsymbol{v}},
$$

where $\delta_{\boldsymbol{v}}$ denotes the Dirac measure on $\mathbb{R}^{m}$ concentrated at $\boldsymbol{v}$. Let us observe that, as $\hbar \rightarrow 0$, the measures $\mu^{\hbar}(|d \xi|)$ converge weakly to the measure

$$
\mu^{0}(|d \boldsymbol{\xi}|):=\frac{1}{(2 \pi)^{m}} w(|\xi|)|d \xi|
$$

Denote by $Y^{0}$ the stationary, isotropic, Gaussian random function on $\mathbb{R}^{m}$ with spectral measure $\mu^{0}(|d \xi|)$. Its covariance kernel is

$$
\mathcal{K}^{0}(\mathbf{x}, \mathbf{y})=\frac{1}{(2 \pi)^{m}} \int_{\mathbb{R}^{m}} e^{-\mathbf{i}\langle\xi, \mathbf{y}-\mathbf{x}\rangle} w(|\xi|)|d \xi|=V(\mathbf{y}-\mathbf{x}) .
$$

From (1.12) and (1.13), we deduce that

$$
\mathcal{K}^{\hbar} \rightarrow \mathcal{K}^{0} \quad \text { in } C^{\infty} \text { as } \hbar \rightarrow 0 .
$$

This suggests that the statistics of $Y^{\hbar}$ ought to be "close" to the statistics of $Y^{0}$. In [32] we showed that the number of critical points of $Y^{0}$ on large cubes of size $\sim \hbar^{-1}$ satisfy a central limit theorem as $\hbar \rightarrow 0$. To prove that a similar result is true for the functions $Y^{\hbar}$ we need to give a white noise description of these random functions.

Recall (see [22], Chapter 7) that a Gaussian white-noise on $\mathbb{R}^{m}$ is characterized by a probability space $(\Omega, \mathcal{F}, \mathbb{P})$ and an isometry $\mathbf{I}=\mathbf{I}_{W}: L^{2}\left(\mathbb{R}^{m}, d \boldsymbol{\xi}\right) \rightarrow L^{2}(\Omega, \mathcal{F}, \mathbb{P})$ onto a Gaussian subspace of $L^{2}(\Omega, \mathcal{F}, \mathbb{P})$. For $f \in L^{2}\left(\mathbb{R}^{m}, d \boldsymbol{\xi}\right)$ the random variable $\mathbf{I}_{W}(f)$ is expressed as an Itô integral

$$
\mathbf{I}_{W}(f)=\int_{\mathbb{R}^{m}} f(\xi) W(d \boldsymbol{\xi})
$$

where $W$ is random measure given by

$$
W(A)=\mathbf{I}_{W}\left[\mathbf{1}_{A}\right], \quad \forall A \in \mathcal{B}\left(\mathbb{R}^{m}\right) .
$$

The existence of Gaussian white noises is a well settled fact [18].

Fix two independent Gaussian white-noises $W_{1}, W_{2}$ on $\mathbb{R}^{m}$ defined on the probability space $(\Omega, \mathcal{F}, \mathbb{P})$. Let $\mathbf{I}_{1}$ and respectively, $\mathbf{I}_{2}$ their associated Itô integrals,

$$
\mathbf{I}_{1}, \mathbf{I}_{2}: L^{2}\left(\mathbb{R}^{m}, d \boldsymbol{\xi}\right) \rightarrow L^{2}(\Omega, \mathcal{F}, \mathbb{P}) .
$$


The independence of the white noises $\mathbf{W}_{1}$ and $\mathbf{W}_{2}$ is equivalent to the condition

$$
\mathbb{E}\left[\mathbf{I}_{1}(f) \mathbf{I}_{2}(g)\right]=0, \quad \forall f, g \in L^{2}\left(\mathbb{R}^{m}, d \xi\right)
$$

This shows that we have a well-defined isometry

$$
\mathbf{I}: \underbrace{L^{2}\left(\mathbb{R}^{m}, d \boldsymbol{\xi}\right) \times L^{2}\left(\mathbb{R}^{m}, d \boldsymbol{\xi}\right)}_{\mathfrak{H}} \rightarrow L^{2}(\Omega, \mathcal{F}, \mathbb{P}), \quad \mathbf{I}\left(f_{1} \oplus f_{2}\right)=\mathbf{I}_{1}\left(f_{1}\right)+\mathbf{I}_{2}\left(f_{2}\right) .
$$

The map I describes an isonormal Gaussian process parametrized by $\mathfrak{H}$. Its image is a Gaussian Hilbert space $\mathscr{X} \subset L^{2}(\Omega, \mathcal{F}, \mathbb{P})$. We will use $\mathbf{I}$ to give alternate descriptions to the functions $Y^{\hbar}$, $\hbar \geq 0$.

For each $\mathbf{k} \in \mathbb{Z}^{m}$, we denote by $C_{\mathbf{k}}$ the cube of size 1 centered at $\mathbf{k}$ that is,

$$
C_{\mathbf{k}}:=\left\{\xi \in \mathbb{R}^{m} ;|\xi-\mathbf{k}|_{\infty} \leq \frac{1}{2}\right\}
$$

For each $\mathbf{x} \in \mathbb{R}^{m}$ and $\hbar>0$, we set

$$
\begin{aligned}
\tilde{Y}^{\hbar}(\mathbf{x}):= & \sum_{\mathbf{k} \in \mathbb{Z}^{m}} \sqrt{w(2 \pi \hbar|\mathbf{k}|)}\left(\cos 2 \pi \hbar\langle\mathbf{k}, \mathbf{x}\rangle \mathbf{I}_{1}\left(\mathbf{1}_{\hbar C_{\mathbf{k}}}\right)+\sin 2 \pi \hbar\langle\mathbf{k}, \mathbf{x}\rangle \mathbf{I}_{2}\left(\mathbf{1}_{\hbar C_{\mathbf{k}}}\right)\right) \\
= & \sum_{\mathbf{k} \in \mathbb{Z}^{m}} \int_{\mathbb{R}^{m}} \sqrt{w(2 \pi \hbar|\mathbf{k}|)} \cos 2 \pi \hbar\langle\mathbf{k}, \mathbf{x}\rangle \mathbf{1}_{\hbar C_{\mathbf{k}}}(\boldsymbol{\xi}) W_{1}(d \boldsymbol{\xi}) \\
& +\sum_{\mathbf{k} \in \mathbb{Z}^{m}} \int_{\mathbb{R}^{m}} \sqrt{w(2 \pi \hbar|\mathbf{k}|)} \sin 2 \pi \hbar\langle\mathbf{k}, \mathbf{x}\rangle \mathbf{1}_{\hbar C_{\mathbf{k}}}(\boldsymbol{\xi}) W_{2}(d \boldsymbol{\xi}) \in \mathscr{X} .
\end{aligned}
$$

The isometry property of $\mathbf{I}$ and (1.8) show that

$$
\mathbb{E}\left[\tilde{Y}^{\hbar}(\mathbf{x}) \tilde{Y}^{\hbar}(\mathbf{y})\right]=\mathcal{K}^{\hbar}(\mathbf{x}, \mathbf{y}) .
$$

Thus, the random function $\tilde{Y}^{\hbar}$ is stochastically equivalent to $Y^{\hbar}$. Next define

$$
\tilde{Y}^{0}(\mathbf{x})=\int_{\mathbb{R}^{m}} \sqrt{w(2 \pi|\xi|)} \cos 2 \pi\langle\boldsymbol{\xi}, \mathbf{x}\rangle W_{1}(d \boldsymbol{\xi})+\int_{\mathbb{R}^{m}} \sqrt{w(2 \pi|\xi|)} \sin 2 \pi\langle\boldsymbol{\xi}, \mathbf{x}\rangle W_{2}(d \boldsymbol{\xi}) \in \mathscr{X}
$$

Then

$$
\begin{aligned}
\mathbb{E}\left[\tilde{Y}^{0}(\mathbf{x}) \tilde{Y}^{0}(\mathbf{y})\right] & =\int_{\mathbb{R}^{m}} w(2 \pi|\xi|) \cos 2 \pi\langle\xi, \mathbf{y}-\mathbf{x}\rangle d \boldsymbol{\xi} \\
& =\frac{1}{(2 \pi)^{m}} \int_{\mathbb{R}^{m}} e^{-\mathbf{i}\langle\boldsymbol{\xi}, \mathbf{y}-\mathbf{x}\rangle} w(|\boldsymbol{\xi}|)|d \boldsymbol{\xi}|=\mathcal{K}^{0}(\mathbf{x}, \mathbf{y})
\end{aligned}
$$

Thus, the random function $\tilde{Y}^{0}$ is stochastically equivalent to $Y^{0}$. 
The above discussion shows that the Gaussian random variables

$$
Y^{\hbar}(\mathbf{x}), \quad \partial_{x_{i}} Y^{\hbar}(\mathbf{x}), \quad \partial_{x_{i} x_{j}}^{2} Y^{\hbar}(\mathbf{x}), \quad 1 \leq i, j \leq m, \mathbf{x} \in \mathbb{R}, \hbar \geq 0,
$$

belong the same Gaussian Hilbert space $\mathscr{X}$. We denote by $\widehat{\mathscr{X}}$ the associated Wiener chaos, $[22,26]$,

$$
\widehat{\mathscr{X}}:=L^{2}(\Omega, \widehat{\mathcal{F}}, \mathbb{P}),
$$

where $\widehat{\mathcal{F}} \subset \mathcal{F}$ is the $\sigma$-algebra generated by the random variables $Z \in \mathscr{X}$.

\subsection{Statements of the main results}

In the sequel, we will use the notation $Q_{\hbar}=O\left(\hbar^{\infty}\right)$ to indicate that, for any $N \in \mathbb{N}$, there exists a constant $C_{N}>0$ such that

$$
\left|Q_{\hbar}\right| \leq C_{N} \hbar^{N} \quad \text { as } \hbar \searrow 0 .
$$

Fix $\hbar_{0}>0$ sufficiently small such that technical Proposition 2.1 holds.

Theorem 1.1. Fix a function $N:\left(0, \hbar_{0}\right) \rightarrow \mathbb{N}, \hbar \mapsto N_{\hbar}$ such that

$$
2 N_{\hbar} \leq \frac{1}{\hbar}, \quad \forall \hbar>0 .
$$

Then, for any box $B \subset \mathbb{R}^{m}$ we have

$$
\begin{aligned}
\mathbb{E}\left[\mathbf{Z}\left(Y^{0}, B\right)\right] & =\bar{Z}_{0}|B|, \quad \bar{Z}_{0}=\frac{1}{\sqrt{\operatorname{det}\left(-2 \pi \nabla^{2} V(0)\right)}} \mathbb{E}\left[\left|\operatorname{det} \nabla^{2} Y^{0}(0)\right|\right], \\
\mathbb{E}\left[\mathbf{Z}\left(Y^{\hbar}, \widehat{B}_{2 N_{\hbar}}\right)\right] & =\mathbb{E}\left[\mathbf{Z}\left(Y^{0}, \widehat{B}_{2 N_{\hbar}}\right)\right]+O\left(\hbar^{\infty}\right) .
\end{aligned}
$$

For simplicity, for any Borel subset $B \subset \mathbb{R}^{m}$, and any $\hbar \in\left[0, \hbar_{0}\right]$ we set

$$
\mathbf{Z}^{\hbar}(B):=\mathbf{Z}\left(Y^{\hbar}, B\right), \quad \zeta^{\hbar}(B)=|B|^{-1 / 2}\left(\mathbf{Z}^{\hbar}(B)-\mathbb{E}\left[\mathbf{Z}^{\hbar}(B)\right]\right) .
$$

For $R>0$, we set

$$
\mathbf{Z}^{\hbar}(R):=\mathbf{Z}^{\hbar}\left(\widehat{B}_{R}\right), \quad \zeta^{\hbar}(R)=\zeta^{\hbar}\left(\widehat{B}_{R}\right)
$$

Theorem 1.2. There exists a number $S^{0}>0$ such that, for any function

$$
N:\left(0, \hbar_{0}\right) \rightarrow \mathbb{N}, \quad \hbar \mapsto N_{\hbar},
$$

satisfying

$$
2 N_{\hbar} \leq \frac{1}{2 \hbar}, \quad \forall \hbar>0,
$$


and

$$
\lim _{\hbar \rightarrow 0} N_{\hbar}=\infty
$$

the following hold.

(i) As $\hbar \rightarrow 0$

$$
\operatorname{var}\left[\mathbf{Z}^{\hbar}\left(2 N_{\hbar}\right)\right] \sim S^{0} \cdot\left(2 N_{\hbar}\right)^{m} \sim \operatorname{var}\left[\mathbf{Z}^{0}\left(2 N_{\hbar}\right)\right] .
$$

(ii) The families of random variables

$$
\left\{\zeta^{\hbar}\left(2 N_{\hbar}\right)\right\}_{\hbar \in\left(0, \hbar_{0}\right]} \text { and }\left\{\zeta^{0}\left(2 N_{\hbar}\right)\right\}_{\hbar \in\left(0, \hbar_{0}\right]}
$$

converge in distribution as $\hbar \rightarrow 0$ to normal random variables $\sim \mathcal{N}\left(0, S^{0}\right)$.

Let us observe that the above theorem works for $N_{\hbar}$ of the form

$$
N_{\hbar}=c\left\lfloor\hbar^{-\alpha}\right\rfloor, \quad 0<\alpha<1, c>0, \hbar \gg 0 .
$$

We believe that for this special choice of $N_{\hbar}$ the result extends to arbitrary noncompact manifolds, not necessarily flat tori.

If $N_{\hbar}$ satisfies $\left(*_{\alpha}\right)$ with $\alpha=1$ the situation is trickier. Recall that for any $r \in(0,1]$ we have denoted by $B_{r} \subset \mathbb{T}^{m}$ the image of $\widehat{B}_{r}$ under the natural projection $\mathbb{R}^{m} \rightarrow \mathbb{T}^{m}$. Note that $B_{1}=\mathbb{T}^{m}$ and recall (1.7),

$$
\mathbf{Z}\left(Y^{\hbar}, \widehat{B}_{\hbar^{-1} r}\right)=\mathbf{Z}\left(X^{\hbar}, B_{r}\right), \quad \forall r \in(0,1] .
$$

If $r$ is fixed in $(0,1 / 2]$, but $\hbar \rightarrow 0$ in such a way that

$$
\frac{r}{2 \hbar}=N_{\hbar} \in \mathbb{N}
$$

then Theorem 1.2 provides an information on the behavior on $\mathbf{Z}\left(X^{\hbar}, B_{r}\right)$ as $\hbar \rightarrow 0$. Our next shows that we reach the same conclusion without assuming the quantization condition $(Q)$.

Theorem 1.3. Let $\bar{Z}_{0}$ be as in $(1.16 a)$ and $S_{0}$ be as in Theorem 1.2. Assume ${ }^{1}$ that

$$
0<r \leq \frac{1}{2}
$$

Then the following hold.

(i) As $\hbar \rightarrow 0$,

$$
\mathbb{E}\left[\mathbf{Z}\left(X^{\hbar}, B_{r}\right)\right]=\hbar^{-m}\left(\bar{Z}_{0} \operatorname{vol}\left(B_{r}\right)+O\left(\hbar^{\infty}\right)\right)
$$

\footnotetext{
${ }^{1}$ The assumption $r \leq 1 / 2$ is the counterpart of $(\$)$.
} 
(ii) As $\hbar \rightarrow 0$ we have

$$
\operatorname{var}\left[\mathbf{Z}\left(X^{\hbar}, B_{r}\right)\right] \sim S_{0} \hbar^{-m} \operatorname{vol}\left(B_{r}\right) .
$$

(iii) As $\hbar \rightarrow 0$ the random variables

$$
\left(\frac{\hbar}{r}\right)^{\frac{m}{2}}\left(\mathbf{Z}\left(X^{\hbar}, B_{r}\right)-\mathbb{E}\left[\mathbf{Z}\left(X^{\hbar}, B_{r}\right)\right]\right)
$$

converge in distribution to a random variable $\sim \mathcal{N}\left(0, S^{0}\right)$.

Corollary 1.4. Let $r \in(0,1 / 2]$. Set

$$
\overline{\mathbf{Z}}^{\hbar}\left(X^{\hbar}, B_{r}\right):=\hbar^{m} \mathbf{Z}\left(X^{\hbar}, B_{r}\right) .
$$

Let $\left(\hbar_{n}\right)$ be a sequence of positive numbers such that, for some $p \in(0, m)$ we have

$$
\sum_{n \geq 1} \hbar_{n}^{p}<\infty
$$

Then

$$
\overline{\mathbf{Z}}^{\hbar_{n}}\left(X^{\hbar_{n}}, B_{r}\right) \rightarrow \bar{Z}_{0} \operatorname{vol}\left(B_{r}\right) \quad \text { a.s. }
$$

Proof. Set $\alpha:=\frac{m-p}{2}$ so that $p=m-2 \alpha$. Note that

$$
\begin{aligned}
\mathbb{E}\left[\overline{\mathbf{Z}}^{\hbar_{n}}\left(X^{\hbar_{n}}, B_{r}\right)\right] & =\bar{Z}_{0} \operatorname{vol}\left(B_{r}\right)+O\left(\hbar_{n}^{\infty}\right), \\
\operatorname{var}\left[\overline{\mathbf{Z}}^{\hbar_{n}}\left(X^{\hbar_{n}}, B_{r}\right)\right] & \sim S_{0} \operatorname{vol}\left(B_{r}\right) \hbar_{n}^{m} .
\end{aligned}
$$

From Chebyshev's inequality, we deduce

$$
\mathbb{P}\left[\left|\overline{\mathbf{Z}}^{\hbar_{n}}\left(X^{\hbar_{n}}, B_{r}\right)-\bar{Z}_{0} \operatorname{vol}\left(B_{r}\right)\right| \geq h_{n}^{\alpha}\right]=O\left(\hbar_{n}^{p}\right) .
$$

Then

$$
\sum_{n \geq 1} \mathbb{P}\left[\left|\overline{\mathbf{Z}}^{\hbar_{n}}\left(X^{\hbar_{n}}, B_{r}\right)-\bar{Z}_{0} \operatorname{vol}\left(B_{r}\right)\right| \geq h_{n}^{\alpha}\right]<\infty,
$$

and the first Borel-Cantelli lemma implies the desired conclusion.

Our final result extends Theorem 1.3 to the degenerate case $r=1$ when the assumption ( $\$)$ is not satisfied.

Theorem 1.5. Let $\bar{Z}_{0}$ be as in (1.16a) and $S_{0}$ be as in Theorem 1.2. Then the following hold.

(i) As $\hbar \rightarrow 0$,

$$
\mathbb{E}\left[\mathbf{Z}\left(X^{\hbar}, \mathbb{T}^{m}\right)\right]=\hbar^{-m}\left(\bar{Z}_{0}+O\left(\hbar^{\infty}\right)\right)
$$


(ii) As $\hbar \rightarrow 0$ we have

$$
\operatorname{var}\left[\mathbf{Z}\left(X^{\hbar}, \mathbb{T}^{m}\right)\right] \sim S_{0} \hbar^{-m}
$$

(iii) As $\hbar \rightarrow 0$ the random variables

$$
\hbar^{\frac{m}{2}}\left(\mathbf{Z}\left(X^{\hbar}, \mathbb{T}^{m}\right)-\mathbb{E}\left[\mathbf{Z}\left(X^{\hbar}, \mathbb{T}^{m}\right)\right]\right)
$$

converge in distribution to a random variable $\sim \mathcal{N}\left(0, S^{0}\right)$.

Remark 1.6. (a) In principle, the method of proof we employ can produce effective bounds on the Fortet-Mourier distance on the space of probability measures on the real axis. For the sake of clarity, we have decided not to pursue this aspect.

(b) Since the random function $X^{\hbar}$ is isotropic with respect to the Abelian group structure on $\mathbb{T}^{m}$, we deduce that Theorem 1.3 applies to cubes in $\mathbb{T}^{m}$ not necessarily centered at 0 .

(c) The constant $\bar{Z}_{0}$ in (1.16a) depends only on $m$ and $w, \bar{Z}_{0}=\bar{Z}_{0}(w, m)$ and represents the expected density of critical points per unit volume of the random function $Y^{0}$. We set

$$
I_{k}(w):=\int_{0}^{\infty} w(r) r^{k} d r
$$

We set

$$
\begin{aligned}
s_{m} & :=\frac{2^{1-\frac{m}{2}}}{\Gamma\left(\frac{m}{2}\right)} I_{m-1}(w), \quad d_{m}:=\frac{2^{1-\frac{m}{2}}}{m \Gamma\left(\frac{m}{2}\right)} I_{m+1}(w), \\
h_{m} & :=\frac{2^{1-\frac{m}{2}}}{m(m+2) \Gamma\left(\frac{m}{2}\right)} I_{m+3}(w) .
\end{aligned}
$$

Denote by $\mathcal{S}_{m}$ the Gaussian Orthogonal Ensemble of real symmetric $m \times m$ matrices $A$ with independent, normally distributed entries $\left(a_{i j}\right)_{1 \leq i, j \leq m}$ with variances

$$
\mathbb{E}\left[a_{i i}^{2}\right]=2, \quad \mathbb{E}\left[a_{i j}^{2}\right]=1, \quad \forall 1 \leq i \neq j \leq m .
$$

As explained in [32], we have

$$
\bar{Z}_{0}(w, m)=\left(\frac{h_{m}}{2 \pi d_{m}}\right)^{\frac{m}{2}} \mathbb{E}_{\mathcal{S}_{m}}[|\operatorname{det} A|]=\left(\frac{I_{m+1}(w)}{2 \pi(m+2) I_{m+3}(w)}\right)^{\frac{m}{2}} \mathbb{E}_{\mathcal{S}_{m}}[|\operatorname{det} A|] .
$$

In [30], Corollary 1.7, we have shown that, as $m \rightarrow \infty$, we have

$$
\bar{Z}_{0}(w, m) \sim \frac{8}{\sqrt{\pi m}} \Gamma\left(\frac{m+3}{2}\right)\left(\frac{2 I_{m+3}(w)}{\pi(m+2) I_{m+1}(w)}\right)^{\frac{m}{2}} .
$$

The asymptotic behavior of $\bar{Z}_{0}(w, m)$ as $m \rightarrow \infty$ depends rather dramatically on the size of the tail of the Schwarz function $w$ : the heavier the tail, the faster the growth of $\bar{Z}_{0}(w, m)$ as $m \rightarrow \infty$. For example, in [30], Section 3, we have shown the following. 
- If $w(t) \sim \exp (-(\log t) \log (\log t))$ as $t \rightarrow \infty$, then

$$
\log \bar{Z}_{0}(w, m) \sim \frac{m}{2} e^{m+2}\left(e^{2}-1\right) \quad \text { as } m \rightarrow \infty .
$$

- If $w(t) \sim \exp \left(-(\log t)^{\frac{p}{p-1}}\right)$ as $t \rightarrow \infty, p>1$, then, for some explicit constant $C_{p}>0$, we have

$$
\log \bar{Z}_{0}(w, m) \sim C_{p} m^{p}, \quad \text { as } m \rightarrow \infty .
$$

- If $w(t) \sim e^{-t^{2}}$ as $t \rightarrow \infty$, then

$$
\log \bar{Z}_{0}(w, m) \sim \sim \frac{m}{2} \log m, \quad \text { as } m \rightarrow \infty .
$$

(d) The constant $S_{0}$ in Theorem 1.2 seems very difficult to estimate. As the proof of Theorem 1.2 will show, the constant $S_{0}$ is a sum of a series with nonnegative terms

$$
S_{0}=\sum_{q \geq 1} \bar{S}_{q}^{0},
$$

where the terms $\bar{S}_{q}^{0}$ are defined explicitly in (2.23). In [32], we have proved that $S_{0}>0$ by showing that

$$
\bar{S}_{2}^{0}=\int_{\mathbb{R}^{m}}\left|P\left(\xi_{1}, \ldots, \xi_{m}\right) w(|\boldsymbol{\xi}|)\right|^{2} d \boldsymbol{\xi},
$$

where $P\left(\xi_{1}, \ldots, \xi_{m}\right)$ is a certain explicit, nonzero, but rather complicated polynomial. The constant $\bar{S}_{2}^{0}$ depends on $w$ and $m$. In [32], Appendix A, we described methods of producing asymptotic estimates for $\bar{S}_{2}^{0}(w, m)$ as $m \rightarrow \infty$, but the results are not too pretty.

\subsection{Outline of proofs}

The strategy of proof is inspired from [10,17]. As explained earlier, the Gaussian random variables $\partial_{\mathbf{x}}^{\alpha} Y^{\hbar}(\mathbf{x}), \mathbf{x} \in \mathbb{R}^{m}, \hbar \geq 0,|\alpha| \leq 2$, are defined on the same probability space $(\Omega, \mathcal{F}, \mathbf{P})$ and belong to the same Gaussian Hilbert space $\mathscr{X}$.

We should point out two major differences between this work and [17]. First of all, the stationary random function $Y^{\hbar}$ on $\mathbb{R}^{m}$ is not isotropic if $\hbar>0$. Moreover, since this function is $\left(\hbar^{-1} \mathbb{Z}\right)^{m}$-periodic, its covariance kernel $V^{\hbar}(\mathbf{z})$ does not decay to 0 as $|\mathbf{z}| \rightarrow \infty$. These are two ingredients that play an important role in [17].

To deal with the lack of isotropy we rely on asymptotic estimates in [31] which provide a precise but rather intricate quantitative meaning to the intuitive observation that $Y^{\hbar}$ is "nearly isotropic for $\hbar$ small" since $Y^{0}$ is isotropic. The fact that $V^{\hbar}(\mathbf{z})$ does not decay at infinity is a nagging issue. We have dealt with it by working with a truncation $(2.15)$ of $V^{\hbar}$ which is the extension by 0 of the restriction of $V^{\hbar}$ to a certain fundamental domain of the lattice $\left(\hbar^{-1} \mathbb{Z}\right)^{m}$. The truncated function (2.15) satisfies the estimates (2.16). This choice forces on us the technical conditions $(\dagger)$ and $(\dagger)$. 
Using the Kac-Rice formula and the asymptotic estimates in [31] we show in Section 2.1 that, for any $\hbar \geq 0$ sufficiently small, and any box $B$, the random variables $\mathbf{Z}^{\hbar}(B)$ belongs to the Wiener chaos $\widehat{\mathscr{X}}$ and we describe its Wiener chaos decomposition. The key result behind this fact is Proposition 2.1 whose rather involved technical proof is deferred to Appendix. The Wiener chaos decomposition of $\mathbf{Z}^{\hbar}(B)$ leads immediately to (1.16a) and (1.16b).

We do not need the massive machinery of the Wiener chaos decomposition to derive these equalities, but since we had to develop it for the proofs of our next results we found it convenient to use it to prove (1.16a) and (1.16b) as well. In [30] we show that Theorem 1.1 holds for arbitrary compact Riemann manifolds.

To prove that the random variables $\zeta^{\hbar}\left(2 N_{\hbar}\right)$ and $\zeta^{0}\left(2 N_{\hbar}\right)$ converge in law to normal random variable $\bar{\zeta}^{0}(\infty)$ and respectively, $\zeta^{0}(\infty)$ we imitate the strategy in [17,32] based on the very general Breuer-Major type central limit theorem [34], Theorem 6.3.1, [33,35-37].

The case of the variables $\zeta^{0}\left(N_{\hbar}\right)$ is covered in [32] where we have shown that there exists $S^{0}>0$ and a normal random variable $\zeta^{0}(\infty) \sim \mathcal{N}\left(0, S^{0}\right)$ such that, as $N \rightarrow \infty$, the random variable $\zeta^{0}(N)$ converges in law to $\zeta^{0}(\infty)$.

The case $\zeta^{\hbar}\left(2 N_{\hbar}\right)$ is conceptually similar, but the extra dependence on $\hbar$ adds an extra layer of difficulty. Here are the details.

Denote by $\zeta_{q}^{\hbar}$ the $q$ th chaos component of $\zeta_{q}^{\hbar}\left(2 N_{\hbar}\right) \in \widehat{\mathscr{X}}$. According to [34], Theorem 6.3.1, to prove that $\zeta^{\hbar}\left(2 N_{\hbar}\right)$ converges in law to a normal random variable $\bar{\zeta}^{0}(\infty)$ it suffices to prove the following.

(i) For every $q \in \mathbb{N}$ there exists $\bar{S}_{q}^{0} \geq 0$ such that

$$
\lim _{\hbar \rightarrow 0} \operatorname{var}\left[\zeta_{q}^{\hbar}\right]=\bar{S}_{q}^{0}
$$

(ii) Exists $\hbar_{0}>0$ such that

$$
\lim _{Q \rightarrow \infty} \sup _{0 \leq \hbar \leq \hbar_{0}} \sum_{q \geq Q} \operatorname{var}\left[\zeta_{q}^{\hbar}\right]=0 .
$$

(iii) For each $q \in \mathbb{N}$, the random variables $\zeta_{q}^{\hbar}\left(2 N_{\hbar}\right)$ converge in law to a normal random variable, necessarily of variance $\bar{S}_{q}^{0}$.

We prove (i) and (ii) in Section 2.4; see (2.24) and respectively, Lemma 2.6.

To prove (iii), we rely on the fourth-moment theorem [34], Theorem 5.2.7, [36]. The details are identical to the ones employed in the proof of [17], Proposition 2.4. The variance of the limiting normal random variable $\bar{\zeta}^{0}(\infty)$ is

$$
\operatorname{var}\left[\bar{\zeta}_{0}(\infty)\right]=\sum_{q \geq 1} \bar{S}_{q}^{0}<\infty .
$$

The explicit description of the components $\bar{S}_{q}^{0}$ will then show that $S^{0}=\bar{S}^{0}$.

We should explain here where the role of the technical assumption (†). In estimating the variance, we need to use the integral formula (2.18) and the estimates (2.16). This is possible only if ( ) is satisfied; see, for example, (2.21). 
The proof of Theorem 1.3 is, up to a suitable rescaling, identical to the proof of Theorem 1.2. We explain this in more detail in Section 2.6.

As we have mentioned earlier, Theorem 1.5 extends Theorem 1.3 to the cube $B_{1}$ that violates the technical condition ( $\ddagger)$. Its proof uses two new ideas. The first is the equality (2.36) which is obtained by subdividing the cube $\widehat{B}_{2} / \hbar$ centered at the origin and with vertices in the lattice $L_{\hbar}=\left(\hbar^{-1} \mathbb{Z}\right)^{m}$ into the sub-cubes $\widehat{B}_{2 / \hbar}^{\omega}$ corresponding to the $2^{m}$ octants of $\mathbb{R}^{m}$. These sub-cubes also have vertices in the same lattice, but what is equally important, the centers of any pair of these sub-cubes differ by a vector in the lattice $L_{\hbar}$ (2.38). To reach the desired conclusion, we need a further decompose the sub-cubes $\widehat{B}_{2 / \hbar}^{\omega}$ into $2^{m}$ cubes of half-size (2.37). The clincher that makes this approach work is the "miraculous" quantization condition (2.38). The details are contained in Section 2.7.

We should point out that the approach we use in the proof of Theorem 1.5 does not apply to cubes of the form $B_{r}, r \in\left(\frac{1}{2}, 1\right)$, that violate $(\ddagger)$. The reason is that the quantization condition (2.38) does not hold in this case.

\subsection{Related results}

Central limit theorems concerning crossing counts of random functions go back a while, for example, Malevich [27] and Cuzik [15].

The usage of Wiener chaos or Hermite decompositions and of Breuer-Major type results [13] in proving such central limit theorems is more recent, late 80 s early $90 \mathrm{~s}$. We want to mention here the pioneering contributions of Chambers and Slud [14], Slud [38,39], Kratz and León [24], Sodin and Tsirelson [40].

This topic was further elaborated by Kratz and León in [25] where they also proved a central limit theorem concerning the length of the zero set of a random function of two variables. We refer to [11] for particularly nice discussion of these developments. Meschenmoser and Shaskin [29] used Hermite decomposition to prove a central limit theorem involving the volumes of level sets of random functions on large cubes in arbitrary dimensions.

Azaiis and León [10] used the technique of Wiener chaos decomposition to give a shorter and more conceptual proof to a central limit theorem due to Granville and Wigman [19] concerning the number of zeros of random trigonometric polynomials of large degree.

In [17], Estrade and León used the Wiener chaos decomposition combined with techniques of Nourdin and Peccati $[33,34]$ to prove a central limit theorem concerning a signed count of critical points of isotropic Gaussian random functions on Euclidean spaces. This approach was then successfully used by Azaiis, Dalmao and León [8] to prove a CLT concerning the number of zeros of Gaussian even trigonometric polynomials and by Dalmao in [16] to prove a CLT concerning the number of zeros of one-variable polynomials in the Kostlan-Shub-Smale probabilistic ensemble. Adler and Naitzat [1] used Hermite decompositions to prove a CLT concerning Euler integrals of random functions.

The recent results [9] suggest that the central limit results proved in this paper may have a universal character in the sense that the random Fourier series (1.5) need not be Gaussian. 


\section{Proofs of the main results}

\subsection{Hermite decomposition of the number of critical points}

For every $\hbar \geq 0, \mathbf{v} \in \mathbb{R}^{m}$ and $B \in \mathcal{B}\left(\mathbb{R}^{m}\right)$ we denote by $\mathbf{Z}^{\hbar}(\mathbf{v}, B)$ the number of solutions $\mathbf{x}$ of the equation

$$
\nabla Y^{\hbar}(\mathbf{x})=\mathbf{v}, \quad \mathbf{x} \in B
$$

For $\varepsilon>0$, we define

$$
\delta_{\varepsilon}: \mathbb{R}^{m} \rightarrow \mathbb{R}, \quad \delta_{\varepsilon}(\mathbf{v})=\varepsilon^{-m} \mathbf{1}_{\widehat{B}_{\varepsilon / 2}(0)}(\mathbf{v}) .
$$

Note that $\delta_{\varepsilon}$ is supported on the cube of size $\varepsilon$ centered at the origin and its total integral is 1 . As $\varepsilon \searrow 0$, the function $\delta_{\varepsilon}$ converges in the sense of distributions to the Dirac $\delta_{0}$. We set

$$
\mathbf{Z}_{\varepsilon}^{\hbar}(\mathbf{v}, B)=\int_{B}\left|\operatorname{det} \nabla^{2} Y^{\hbar}(\mathbf{x})\right| \delta_{\varepsilon}\left(\nabla Y^{\hbar}(\mathbf{x})-\mathbf{v}\right) d \mathbf{x}, \quad \mathbf{Z}^{\hbar}(B):=\left.\mathbf{Z}^{\hbar}(\mathbf{v}, B)\right|_{\mathbf{v}=0} .
$$

We define a box in $\mathbb{R}^{m}$ to be a set $B \subset \mathbb{R}^{m}$ of the form

$$
B=\left[a_{1}, b_{1}\right] \times \cdots \times\left[a_{m}, b_{m}\right], \quad a_{1}<b_{1}, \ldots, a_{m}<b_{m} .
$$

If $B \subset \mathbb{R}^{m}$ is a box [2], Theorem 11.3.1, we deduce that $X$ is a.s. a Morse function on $T$ and in particular, for any $\mathbf{v} \in \mathbb{R}^{m}$, the equation $\nabla X^{\hbar}(\mathbf{x})=\mathbf{v}$ almost surely has no solutions $\mathbf{x} \in \partial B$.

The proof of the Kac-Rice formula [2], Theorem 11.2.3, shows that $\mathbf{Z}^{\hbar}(\mathbf{v}, B) \in L^{1}(\Omega)$ and

$$
\mathbf{Z}_{\varepsilon}^{\hbar}(\mathbf{v}, B) \rightarrow \mathbf{Z}^{\hbar}(\mathbf{v}, B) \quad \text { a.s. as } \varepsilon \rightarrow 0 .
$$

Proposition 2.1. There exists $\hbar_{0}>0$, sufficiently small, such that, the Gaussian random vectors

$$
\nabla Y^{\hbar}(\mathbf{x}), \quad \nabla Y^{\hbar}(\mathbf{x}) \oplus Y^{\hbar}(\mathbf{y}), \quad \mathbf{x}, \mathbf{y} \in \mathbb{R}^{m}, \quad \mathbf{x} \neq \equiv \mathbf{y} \bmod \left(\hbar^{-1} \mathbb{Z}\right)^{m}, \quad \hbar \in\left[0, \hbar_{0}\right],
$$

are nondegenerate, for any $\hbar \in\left[0, \hbar_{0}\right]$ and any box $B \subset \widehat{B}_{2}(0)$, the following hold.

(i) For any $\mathbf{v} \in \mathbb{R}^{m}, \mathbf{Z}^{\hbar}(\mathbf{v}, B) \in L^{2}(\Omega, \widehat{\mathcal{F}}, \mathbb{P})$.

(ii) The function

$$
\mathbb{R}^{m} \ni \mathbf{v} \mapsto \mathbb{E}\left[\mathbf{Z}^{\hbar}(\mathbf{v}, B)^{2}\right] \in \mathbb{R}
$$

is continuous.

(iii) For any $\mathbf{v} \in \mathbb{R}^{m}$

$$
\lim _{\varepsilon \rightarrow 0} \mathbf{Z}_{\varepsilon}^{\hbar}(\mathbf{v}, B)=\mathbf{Z}^{\hbar}(\mathbf{v}, B) \quad \text { in } L^{2}(\Omega) .
$$

(iv) The function

$$
\left[0, \hbar_{0}\right] \ni \hbar \mapsto \mathbf{Z}^{\hbar}(B) \in L^{2}(\Omega, \widehat{\mathcal{F}}, \mathbb{P})
$$

is continuous. 
We defer the proof of Proposition 2.1 to the Appendix. The case $\hbar=0$ of this proposition is discussed in [17], Proposition 1.1. That proof uses in an essential fashion the isotropy of the random function $Y^{0}$. For example, the isotropy implies that the covariance matrix of the Gaussian random vector $\nabla Y^{0}(\mathbf{x})$ is a scalar multiple of the identity for any $\mathbf{x}$. This is no longer the case with the random functions $Y^{\hbar}, \hbar>00$, which are not isotropic, but they are "nearly" so for $\hbar$ small. However, the proof in the "nearly" isotropic case is substantially more elaborated and requires considerable input from our earlier work [31].

Since for any Borel set $B \subset \mathbb{R}^{m}$, and any $\varepsilon>0$ the random variables $\mathbf{Z}_{\varepsilon}^{\hbar}(\mathbf{v}, B)$ belong to the Wiener chaos $\widehat{\mathscr{X}}$ defined in (1.15), we deduce from Proposition 2.1(iii) that, for any $\hbar \leq \hbar_{0}$, and any box $B \subset \widehat{B}_{1 / \hbar}$, the number of critical points $\mathbf{Z}^{\hbar}(B)$ belongs to the Wiener chaos $\widehat{\mathscr{X}}$.

Fix $\hbar_{0}$ as in Proposition 2.1. Consider the random field

$$
\widehat{\mathbf{Y}}^{\hbar}(\mathbf{x}):=\nabla Y^{\hbar}(\mathbf{x}) \oplus \nabla^{2} Y^{\hbar}(\mathbf{x}), \quad \mathbf{x} \in \mathbb{R}^{m}, \hbar \in\left[0, \hbar_{0}\right] .
$$

of dimension

$$
D=m+v(m), \quad v(m):=\frac{m(m+2)}{2} .
$$

For any function $f: \mathbb{R}^{m} \rightarrow \mathbb{R}$ and any $i, j, k \in\{1, \ldots, m\}$ we set

$$
f_{i, j}(\mathbf{x})=\partial_{x_{i}, x_{j}}^{2}, \quad f_{i j, k}=\partial_{x_{i}, x_{j} x_{k}}^{3} f(\mathbf{x}) \quad \text { etc. }
$$

Note that

$$
\mathbb{E}\left[Y_{i}^{\hbar}(\mathbf{x}) Y_{j, k}^{\hbar}(\mathbf{x})\right]=-V_{i, j, k}^{\hbar}(0)=0,
$$

since $V^{\hbar}(\mathbf{x})$ is an even function. Hence, the two components of $\widehat{\mathbf{Y}}^{\hbar}$ are independent. We can find invertible matrices $\Lambda_{1}^{\hbar}$ and $\Lambda_{2}^{\hbar}$ of dimensions $m \times m$ and respectively, $v(m) \times v(m)$, that depend continuously on $\hbar \in\left[0, \hbar_{0}\right]$ such that the probability distributions of the random vectors

$$
U(\mathbf{x})=\left(\Lambda_{1}^{\hbar}\right)^{-1} \nabla Y^{\hbar}(\mathbf{x}) \in \mathbb{R}^{m}, \quad A(\mathbf{x}):=\left(\Lambda_{2}^{\hbar}\right)^{-1} \nabla^{2} Y^{\hbar}(\mathbf{x}) \in \mathbb{R}^{v(m)}
$$

are the canonical Gaussian measures on the Euclidean spaces $\mathbb{R}^{m}$ and $\mathbb{R}^{v(m)}$, respectively. More precisely, we can choose as $\Lambda_{i}^{\hbar}, i=1,2$, the square roots of the covariance matrices of $\nabla^{i} Y^{\hbar}(\mathbf{x})$. $\left(\right.$ Here $\left.\nabla^{1}=\nabla.\right)$

The random vectors $U(\mathbf{x})$ and $A(\mathbf{x})$ do depend on $\hbar$ but their probability distributions do not. To ease the notational burden we chose not to include the $\hbar$-dependency in the notation.

Consider the functions

$$
\begin{array}{rlrl}
f^{\hbar}: \mathbb{R}^{v(m)} & \rightarrow \mathbb{R}, \quad & f^{\hbar}(A)=\left|\operatorname{det} \Lambda_{2}^{\hbar} A\right|, \\
G_{\varepsilon}^{\hbar}: \mathbb{R}^{m} \times \mathbb{R}^{v(m)} \rightarrow \mathbb{R}, & G_{\varepsilon}^{\hbar}(U, A)=\delta_{\varepsilon}\left(\Lambda_{1}^{\hbar} U\right) f_{\hbar}(A) .
\end{array}
$$

Fix a box $B$, independent of $\hbar$. Proposition 2.1 shows that, for $\hbar$ sufficiently small, we have

$$
\mathbf{Z}^{\hbar}(B)=\lim _{\varepsilon \rightarrow 0} \int_{B} G_{\varepsilon}^{\hbar}(U(\mathbf{x}), A(\mathbf{x})) .
$$


Recall that an orthogonal basis of $L^{2}(\mathbb{R}, \boldsymbol{\gamma}(d x))$ is given by the Hermite polynomials, [22], Example 3.18, [28], V.1.3,

$$
H_{n}(x):=(-1)^{n} e^{\frac{x^{2}}{2}} \frac{d^{n}}{d x^{n}}\left(e^{-\frac{x^{2}}{2}}\right)=n ! \sum_{r=0}^{\left\lfloor\frac{n}{2}\right\rfloor} \frac{(-1)^{r}}{2^{r} r !(n-2 r) !} x^{n-2 r}
$$

In particular,

$$
H_{n}(0)= \begin{cases}0, & n \equiv 1 \bmod 2, \\ (-1)^{r} \frac{(2 r) !}{2^{r} r !}, & n=2 r .\end{cases}
$$

For every multi-index $\alpha=\left(\alpha_{1}, \alpha_{2}, \ldots\right) \in \mathbb{N}_{0}^{\mathbb{N}}$ such that all but finitely many $\alpha_{k}$-s are nonzero, and any

$$
\underline{x}=\left(x_{1}, x_{2}, \ldots\right) \in \mathbb{R}^{\mathbb{N}}
$$

we set

$$
|\alpha|:=\sum_{k} \alpha_{k}, \quad \alpha !:=\prod_{k} \alpha_{k} !, \quad H_{\alpha}(\underline{x}):=\prod_{k} H_{\alpha_{k}}\left(x_{k}\right) .
$$

Following [17], equation (5), we define for every $\alpha \in \mathbb{N}_{0}^{m}$ the quantity

$$
d_{\alpha}:=\frac{1}{\alpha !}(2 \pi)^{-\frac{m}{2}} H_{\alpha}(0)
$$

The function $f^{\hbar}: \mathbb{R}^{v(m)} \rightarrow \mathbb{R}$ has a $L^{2}\left(\mathbb{R}^{v(m)}, \Gamma\right)$-orthogonal decomposition

$$
f^{\hbar}(A)=\sum_{n \geq 0} f_{n}^{\hbar}(A)
$$

where

$$
f_{n}^{\hbar}(A)=\sum_{\substack{\beta \in \mathbb{N}_{0}^{v(m)} \\|\beta|=n}} f_{\beta}^{\hbar} H_{\beta}(A), \quad f_{\beta}^{\hbar}=\frac{1}{\beta !} \int_{\mathbb{R}^{v(m)}} f^{\hbar}(A) H_{\beta}(A) \Gamma(d A) .
$$

Note that

$$
f_{0}^{\hbar}=\mathbb{E}\left[\left|\operatorname{det} \nabla^{2} Y^{\hbar}(0)\right|\right]
$$

The function $\delta_{\varepsilon}(U)$ has an $L^{2}\left(\mathbb{R}^{m}, \Gamma\right)$-orthogonal decomposition

$$
\delta_{\varepsilon}(U)=\sum_{\alpha \in \mathbb{N}_{0}^{m}} d_{\alpha, \varepsilon}^{\hbar} H_{\alpha}(U)
$$

where

$$
d_{\alpha, \varepsilon}^{\hbar}=\frac{1}{\alpha !} \int_{\mathbb{R}} \delta_{\varepsilon}\left(\Lambda_{1}^{\hbar} U\right) H_{\alpha}(U) \Gamma(d U)
$$


Note that

$$
\lim _{\varepsilon \rightarrow 0} \int_{\mathbb{R}} \delta_{\varepsilon}\left(\Lambda_{1}^{\hbar} U\right) H_{\alpha}(U) \Gamma(d U)=\frac{1}{\operatorname{det} \Lambda_{1}^{\hbar}} H_{\alpha}(0),
$$

so that

$$
\lim _{\varepsilon \rightarrow 0} d_{\alpha, \varepsilon}^{\hbar}=\frac{1}{\operatorname{det} \Lambda_{1}^{\hbar}} d_{\alpha}
$$

uniformly for $\hbar \in\left[0, \hbar_{0}\right]$. We set

$$
\omega_{\hbar}:=\frac{1}{\operatorname{det} \Lambda_{1}^{\hbar}}
$$

Remark 2.2. The matrix $\Lambda_{1}^{\hbar}$ is the square root of the covariance matrix of the random vector $\nabla Y^{\hbar}(0)$, that is,

$$
\Lambda_{1}^{\hbar}=\sqrt{-\nabla^{2} V^{\hbar}(0)}
$$

The function $V=V^{\hbar=0}$ is radially symmetric and thus

$$
\nabla^{2} V(0)=-\lambda^{2} \mathbb{1}_{m}
$$

for some $\lambda>0$. Hence,

$$
\Lambda_{1}^{0}=\lambda \mathbb{1}_{m}, \quad \omega_{0}=\lim _{\hbar \rightarrow 0} \omega_{\hbar}=\lambda^{-m}=\frac{1}{\sqrt{\operatorname{det}\left(-\nabla^{2} V(0)\right)}} .
$$

If we set

$$
\mathcal{J}_{m}:=\mathbb{N}_{0}^{m} \times \mathbb{N}_{0}^{v(m)}
$$

Then

$$
\mathbf{Z}_{\varepsilon}^{\hbar}(B)=\sum_{q=0}^{\infty} \int_{B} \rho_{q, \varepsilon}^{\hbar}(\mathbf{x}) d \mathbf{x}
$$

where

$$
\rho_{q, \varepsilon}^{\hbar}(\mathbf{x})=\sum_{\substack{(\alpha, \beta) \in \mathcal{J}_{m},|\alpha|+|\beta|=q}} d_{\alpha, \varepsilon}^{\hbar} f_{\beta}^{\hbar} H_{\alpha}(U(\mathbf{x})) H_{\beta}(A(\mathbf{x})) .
$$

If we let $\varepsilon \rightarrow 0$ in (2.8) and use Proposition 2.1(iii) and (2.6), we deduce

$$
\begin{aligned}
Z^{\hbar}(B) & =\sum_{q \geq 0} Z_{q}^{\hbar}(B), \quad Z_{q}^{\hbar}(B)=\int_{B} \rho_{q}^{\hbar}(\mathbf{x}) d b x, \\
\rho_{q}^{\hbar}(\mathbf{x}) & =\sum_{\substack{(\alpha, \beta) \in \mathcal{J}_{m},|\alpha|+|\beta|=q}} \omega_{\hbar} d_{\alpha} f_{\beta}^{\hbar} H_{\alpha}(U(\mathbf{x})) H_{\beta}(A(\mathbf{x})) .
\end{aligned}
$$

To proceed further, we need to use some basic Gaussian estimates. 


\subsection{A technical interlude}

Let $\mathbf{V}$ be a real Euclidean space of dimension $N$. We denote by $\mathcal{A}(\mathbf{V})$ the space of symmetric positive semidefinite operators $A: \mathbf{V} \rightarrow \mathbf{V}$. For $A \in \mathcal{A}(\mathbf{V})$, we denote by $\boldsymbol{\gamma}_{A}$ the centered Gaussian measure on $\mathbf{V}$ with covariance form $A$. Thus

$$
\boldsymbol{\gamma}_{\mathbb{1}}(d \mathbf{v})=\frac{1}{(2 \pi)^{\frac{N}{2}}} e^{-\frac{1}{2}|\mathbf{v}|^{2}} d \mathbf{v},
$$

and $\boldsymbol{\gamma}_{A}$ is the push forward of $\boldsymbol{\gamma}_{\mathbb{1}}$ via the linear map $\sqrt{A}$,

$$
\boldsymbol{\gamma}_{A}=(\sqrt{A})_{*} \boldsymbol{\gamma}_{\mathbb{1}}
$$

For any measurable $f: \mathbf{V} \rightarrow \mathbb{R}$ with at most polynomial growth, we set

$$
\mathbb{E}_{A}(f):=\int_{\mathbf{V}} f(\mathbf{v}) \boldsymbol{\gamma}_{A}(d \mathbf{v}) .
$$

We will need the following technical result. For a proof, we refer to [31], Appendix A.

Proposition 2.3. Let $f: \mathbf{V} \rightarrow \mathbb{R}$ be a locally Lipschitz function which is positively homogeneous of degree $\alpha \geq 1$. Denote by $L_{f}$ the Lipschitz constant of the restriction of $f$ to the unit ball of $\mathbf{V}$. There exists a constant $C>0$ which depends only on $N$ and $\alpha$ such that, for any $\Lambda>0$ and any $A, B \in \mathcal{A}(\mathbf{V})$ such that $\|A\|,\|B\| \leq \Lambda$ we have

$$
\left|\mathbb{E}_{A}(f)-\mathbb{E}_{B}(f)\right| \leq C L_{f} \Lambda^{\frac{\alpha-1}{2}}\|A-B\|^{\frac{1}{2}}
$$

\subsection{Proof of Theorem 1.1}

Note that

$$
\mathbb{E}\left[\mathbf{Z}^{\hbar}(B)\right]=\mathbb{E}\left[Z_{0}^{\hbar}(B)\right]=|B| \omega_{\hbar} f_{0}^{\hbar} d(0)
$$

(use (2.3) and (2.5))

$$
=(2 \pi)^{-m / 2}|B| \omega_{\hbar} \mathbb{E}\left[\left|\operatorname{det} \nabla^{2} Y^{\hbar}(0)\right|\right] .
$$

Using (1.12) and Remark 2.2, we deduce that

$$
\omega_{\hbar}-\omega_{0}=O\left(\hbar^{\infty}\right) .
$$

The estimate (1.12) implies that

$$
\left\|\nabla^{2} V^{\hbar}(0)-\nabla^{2} V(0)\right\|=O\left(\hbar^{\infty}\right) .
$$


Invoking Proposition 2.3, we deduce that

$$
\mathbb{E}\left[\left|\operatorname{det} \nabla^{2} Y^{\hbar}(0)\right|\right]=\mathbb{E}\left[\left|\operatorname{det} \nabla^{2} Y^{0}(0)\right|\right]+O\left(\hbar^{\infty}\right) .
$$

Hence,

$$
\begin{aligned}
& \mathbb{E}\left[\mathbf{Z}^{\hbar}(B)\right]=\mathbb{E}\left[\mathbf{Z}^{0}(B)\right]+O\left(\hbar^{\infty}\right), \\
& \mathbb{E}\left[\mathbf{Z}^{0}(B)\right]=(2 \pi)^{-\frac{m}{2}} \omega_{0}|B| \mathbb{E}\left[\left|\operatorname{det} \nabla^{2} Y^{0}(0)\right|\right]
\end{aligned}
$$

Using (2.7) in the above equality, we obtain (1.16a).

Let $N_{\hbar}$ satisfy $(\dagger)$. Recall that $\mathbb{A}^{m}$ denotes the affine lattice

$$
\mathbb{A}^{m}=\left(\frac{1}{2}+\mathbb{Z}\right)^{m}
$$

We have

$$
\widehat{B}_{2 N_{\hbar}}=\bigcup_{\mathbf{a} \in \mathbb{A}^{m},|\mathbf{a}|_{\infty} \leq N_{\hbar}} \widehat{B}(\mathbf{a}), \quad \widehat{B}(a):=\widehat{B}_{1}(\mathbf{a}) .
$$

The cubes in the above union have disjoint interiors. According to [2], Theorem 11.3.1, for $\hbar \leq \hbar_{0}$ the function $Y^{\hbar}$ is a.s. Morse. Given a box $B \subset \mathbb{R}^{m}$, the function $Y^{\hbar}$ will a.s. have no critical points on the boundary of $B$. Thus,

$$
\mathbf{Z}^{\hbar}\left(\widehat{B}_{2 N_{\hbar}}\right)=\sum_{\mathbf{a} \in \mathbb{A}^{m} \cap \widehat{B}_{2 N_{\hbar}}} \mathbf{Z}^{\hbar}(\widehat{B}(\mathbf{a}))
$$

From $(\dagger)$, we deduce that $\widehat{B}(\mathbf{a}) \subset \widehat{B}_{1 / \hbar}(0)$ so (1.13) holds on $\widehat{B}(\mathbf{a})$. We deduce

$$
\mathbf{Z}^{\hbar}\left(\widehat{B}_{2 N_{\hbar}}\right) \stackrel{(2.12)}{=} \sum_{\mathbf{a} \in \mathbb{A}^{m} \cap \widehat{B}_{2 N_{\hbar}}}\left(\mathbf{Z}^{0}(\widehat{B}(\mathbf{a}))+O\left(\hbar^{\infty}\right)\right)
$$

From $(\dagger)$, we deduce that $2 N_{\hbar} \leq \frac{1}{\hbar}$ so that

$$
\#\left(\mathbf{a} \in \mathbb{A}^{m} \cap \widehat{B}_{2 N_{\hbar}}\right)=O\left(\hbar^{-m}\right) .
$$

Hence,

$$
\mathbf{Z}^{\hbar}\left(\widehat{B}_{2 N_{\hbar}}\right)=\left(\sum_{\mathbf{a} \in \mathbb{A}^{m} \cap \widehat{B}_{2 N_{\hbar}}} \mathbf{Z}^{0}(\widehat{B}(\mathbf{a}))\right)+O\left(\hbar^{\infty}\right)=\mathbf{Z}^{0}\left(\widehat{B}_{2 N_{\hbar}}\right)+O\left(\hbar^{\infty}\right)
$$




\subsection{Variance estimates}

For $\hbar \in\left[0, \hbar_{0}\right]$, we define

$$
\psi^{\hbar}: \mathbb{R}^{m} \rightarrow \mathbb{R}, \quad \psi^{\hbar}(\mathbf{x})= \begin{cases}\max _{|\alpha| \leq 4}\left|\partial_{\mathbf{x}}^{\alpha} V^{\hbar}(\mathbf{x})\right|, & |\mathbf{x}|_{\infty} \leq \frac{1}{2 \hbar}, \\ 0, & |\mathbf{x}|_{\infty}>\frac{1}{2 \hbar}\end{cases}
$$

Lemma 2.4. For any $p \in[0, \infty]$ we have

$$
\left\|\psi^{\hbar}-\psi^{0}\right\|_{L^{p}\left(\mathbb{R}^{m}\right)}=O\left(\hbar^{\infty}\right) .
$$

Proof. We distinguish two cases.

1. $p=\infty$. Note that (1.13) implies

$$
\sup _{|\mathbf{x}|_{\infty} \leq 1 /(2 \hbar)}\left|\psi^{\hbar}(\mathbf{x})-\psi^{0}(\mathbf{x})\right|=O\left(\hbar^{\infty}\right) .
$$

Since $V$ is a Schwarz function we deduce that

$$
\sup _{|\mathbf{x}|_{\infty}>1 /(2 \hbar)}\left|\psi^{\hbar}(\mathbf{x})-\psi^{0}(\mathbf{x})\right|=\sup _{|\mathbf{x}|_{\infty}>1 /(2 \hbar)}\left|\psi^{0}(\mathbf{x})\right|=O\left(\hbar^{\infty}\right) .
$$

2. $p \in[1, \infty)$. We have

$$
\int_{\mathbb{R}^{m}}\left|\psi^{\hbar}(\mathbf{x})-\psi^{0}(\mathbf{x})\right|^{p} d \mathbf{x}=\int_{|\mathbf{x}|_{\infty} \leq 1 /(2 \hbar)}\left|\psi^{\hbar}(\mathbf{x})-\psi^{0}(\mathbf{x})\right|^{p} d \mathbf{x}+\int_{|\mathbf{x}|_{\infty}>1 /(2 \hbar)}\left|\psi^{0}(\mathbf{x})\right|^{p} d \mathbf{x}
$$

The integrand in the first integral in the right-hand side is $O\left(\hbar^{\infty}\right)$ and the volume of the region is integration is $O\left(\hbar^{-m}\right)$ so the first integral is $O\left(\hbar^{\infty}\right)$. Since $V$ is a Schwarz function, we deduce that

$$
\left|\psi^{0}(\mathbf{x})\right|=O\left(|\mathbf{x}|^{-N}\right), \quad \forall N \in \mathbb{N} .
$$

This shows that the second integral is also $O\left(\hbar^{\infty}\right)$.

Proposition 2.5. There exists $S^{0} \in(0, \infty)$ such that

$$
\lim _{h \searrow 0} \operatorname{var}\left[\zeta^{\hbar}\left(2 N_{\hbar}\right)\right]=S^{0}=\lim _{h \searrow 0} \operatorname{var}\left[\zeta^{0}\left(2 N_{\hbar}\right)\right] .
$$

Proof. In [32], we proved that the limit

$$
\lim _{N \rightarrow \infty} \operatorname{var}\left[\zeta^{0}(2 N)\right]
$$

exists, it is finite and nonzero. We denote by $S^{0}$ this limit. It remains to prove two facts. 
(F $\left.\mathbf{F}_{1}\right)$ The limit $\bar{S}^{0}:=\lim _{h \searrow 0} \operatorname{var}\left[\zeta^{\hbar}\left(2 N_{\hbar}\right)\right]$ exists and it is finite.

(F) $S^{0}=\bar{S}^{0}$.

To prove these facts, we will employ a refinement of the strategy used in the proof of [32], Proposition 3.3.

Proof of $\mathbf{F}_{1}$. Using (2.9a), we deduce

$$
\zeta^{\hbar}\left(2 N_{\hbar}\right)=\left(2 N_{\hbar}\right)^{-m / 2}\left(\mathbf{Z}^{\hbar}\left(\widehat{B}_{2 N_{\hbar}}\right)-\mathbb{E}\left[\mathbf{Z}^{\hbar}\left(\widehat{B}_{2 N_{\hbar}}\right)\right]\right)=\left(2 N_{\hbar}\right)^{-m / 2} \sum_{q>0} Z_{q}^{\hbar}\left(\widehat{B}_{2 N_{\hbar}}\right) .
$$

We set

$$
S^{\hbar}:=\operatorname{var}\left[\zeta^{\hbar}\left(2 N_{\hbar}\right)\right]=\mathbb{E}\left[\zeta^{\hbar}\left(2 N_{\hbar}\right)^{2}\right]=\sum_{q>0} \underbrace{\left(2 N_{\hbar}\right)^{-m} \mathbb{E}\left[Z^{\hbar}\left(\widehat{B}_{2 N_{\hbar}}\right)^{2}\right]}_{=: S_{q}^{\hbar}} .
$$

To estimate $S_{q}^{\hbar}$, we write

$$
Z_{q}^{\hbar}\left(\widehat{B}_{2 N_{\hbar}}\right)=\int_{\widehat{B}_{2 N_{\hbar}}} \rho_{q}^{\hbar}(\mathbf{x}) d \mathbf{x}
$$

where $\rho_{q}^{\hbar}(\mathbf{x})$ is described in (2.9b). Then

$$
S_{q}^{\hbar}=\left(2 N_{\hbar}\right)^{-m} \int_{\widehat{B}_{2 N_{\hbar}} \times \widehat{B}_{2 N_{\hbar}}} \mathbb{E}\left[\rho_{q}^{\hbar}(\mathbf{x}) \rho_{q}^{\hbar}(\mathbf{y})\right] d \mathbf{x} d \mathbf{y} .
$$

Now use the stationarity of $Y^{\hbar}(\mathbf{x})$ to conclude

$$
\begin{aligned}
& =\left(2 N_{\hbar}\right)^{-m} \int_{\widehat{B}_{2 N_{\hbar}} \times \widehat{B}_{2 N_{\hbar}}} \mathbb{E}\left[\rho_{q}^{\hbar}(0) \rho_{q}^{\hbar}(\mathbf{y}-\mathbf{x})\right] d \mathbf{x} d \mathbf{y} \\
& =\int_{\widehat{B}_{4 N_{\hbar}}} \mathbf{E}\left[\rho_{q}^{\hbar}(0) \rho_{q}^{\hbar}(\mathbf{u})\right] \prod_{k=1}^{m}\left(1-\frac{\left|u_{k}\right|}{2 N_{\hbar}}\right) d \mathbf{u} .
\end{aligned}
$$

The last equality is obtained by integrating along the fibers of the map

$$
\widehat{B}_{2 N_{\hbar}} \times \widehat{B}_{2 N_{\hbar}} \ni(\mathbf{x}, \mathbf{y}) \mapsto \mathbf{y}-\mathbf{x} \in \widehat{B}_{4 N_{\hbar}} .
$$

At this point, we need to invoke (2.9b) to the effect that

$$
\rho_{q}^{\hbar}(\mathbf{x})=\sum_{\substack{(\alpha, \beta) \in \mathcal{J}_{m},|\alpha|+|\beta|=q}} \omega_{\hbar} d_{\alpha} f_{\beta}^{\hbar} H_{\alpha}(U(\mathbf{x})) H_{\beta}(A(\mathbf{x}))
$$

We can rewrite this in a more compact form. Set

$$
\Xi^{\hbar}(\mathbf{x}):=(U(\mathbf{x}), A(\mathbf{x})) .
$$


For $\gamma=(\alpha, \beta) \in \mathcal{J}_{m}$ we set

$$
\mathbf{a}^{\hbar}(\gamma):=\omega_{\hbar} d_{\alpha} f_{\beta}^{\hbar}, \quad H_{\gamma}\left(\Xi^{\hbar}(\mathbf{x})\right):=H_{\alpha}(U(\mathbf{x})) H_{\beta}(A(\mathbf{x})) .
$$

Then

$$
\begin{aligned}
& \rho_{q}^{\hbar}(\mathbf{x})=\sum_{\gamma \in \mathcal{J}_{m},|\gamma|=q} \mathbf{a}^{\hbar}(\gamma) H_{\gamma}\left(\Xi^{\hbar}(\mathbf{x})\right), \\
& \mathbb{E}\left[\rho_{q}^{\hbar}(0) \rho_{q}^{\hbar}(u)\right]=\sum_{\substack{\gamma, \gamma^{\prime} \in \mathcal{J}_{m} \\
|\gamma|=\left|\gamma^{\prime}\right|=q}} \mathbf{a}^{\hbar}(\gamma) \mathbf{a}^{\hbar}\left(\gamma^{\prime}\right) \mathbb{E}\left[H_{\gamma}\left(\Xi^{\hbar}(0)\right) H_{\gamma^{\prime}}\left(\Xi^{\hbar}(\mathbf{u})\right)\right] .
\end{aligned}
$$

We set $\omega(m):=m+v(m)$, and we denote by $\boldsymbol{\Xi}_{i}(\mathbf{x}), 1 \leq i \leq \omega(m)$, the components of $\boldsymbol{\Xi}(\mathbf{x})$ labelled so that $\Xi_{i}(\mathbf{x})=U_{i}(\mathbf{x}), \forall 1 \leq i \leq m$. For $\mathbf{u} \in \mathbb{R}^{m}, \hbar \in\left[0, \hbar_{0}\right]$ and $1 \leq i, j \leq \omega(m)$, we define the covariances

$$
\Gamma_{i j}^{\hbar}(\mathbf{u}):=\mathbb{E}\left[\Xi_{i}^{\hbar}(0) \Xi_{j}^{\hbar}(\mathbf{u})\right]
$$

Using the Diagram Formula (see, e.g., [22], Theorem 7.33, or [26], Corollary 5.5) we deduce that for any $\gamma, \gamma^{\prime} \in \mathcal{J}_{m}$ such that $|\gamma|=\left|\gamma^{\prime}\right|=q$, there exists a universal homogeneous polynomial of degree $q, P_{\gamma, \gamma^{\prime}}$ in the variables $\Gamma_{i j}^{\hbar}(\mathbf{u})$ such that

$$
\mathbb{E}\left[H_{\gamma}\left(\Xi^{\hbar}(0)\right) H_{\gamma^{\prime}}\left(\Xi^{\hbar}(\mathbf{u})\right)\right]=P_{\gamma, \gamma^{\prime}}\left(\Gamma_{i j}^{\hbar}(\mathbf{u})\right)
$$

Hence,

$$
S_{q}^{\hbar}=\left(2 N_{\hbar}\right)^{-m} \sum_{\substack{\gamma, \gamma^{\prime} \in \mathcal{J}_{m} \\|\gamma|=\left|\gamma^{\prime}\right|=q}} \mathbf{a}^{\hbar}(\gamma) \mathbf{a}^{\hbar}\left(\gamma^{\prime}\right) \underbrace{\int_{\widehat{B}_{4 N_{\hbar}}} P_{\gamma, \gamma^{\prime}}\left(\Gamma_{i j}^{\hbar}(\mathbf{u})\right) \prod_{k=1}^{m}\left(1-\frac{\left|u_{k}\right|}{2 N_{\hbar}}\right) d \mathbf{u}}_{=: R^{\hbar}\left(\boldsymbol{\gamma}, \boldsymbol{\gamma}^{\prime}\right)} .
$$

From ( $\ddagger)$, we deduce that

$$
\widehat{B}_{4 N_{\hbar}} \subset \widehat{B}_{1 / \hbar}
$$

so that $\widehat{B}_{4 N_{\hbar}} \subset \operatorname{supp} \psi^{\hbar}$, where $\psi^{\hbar}$ is the function defined in (2.15). We deduce that there exists a positive constant $K$, independent of $\hbar \in\left[0, \hbar_{0}\right]$, such that

$$
\left|\Gamma_{i, j}^{\hbar}(\mathbf{u})\right| \leq K \psi^{\hbar}(\mathbf{u}), \quad \forall i, j=1, \ldots, \omega(m), \mathbf{u} \in \widehat{B}_{4 N_{\hbar}} .
$$

From (2.22), we deduce that for any $\gamma, \gamma^{\prime} \in \mathcal{J}_{m}$ such that $|\gamma|=\left|\gamma^{\prime}\right|=q$ there exists a constant $C_{\gamma, \gamma^{\prime}}>0$ such that

$$
\left|P_{\gamma, \gamma^{\prime}}\left(\Gamma_{i j}^{\hbar}(\mathbf{u})\right)\right| \leq C_{\gamma, \gamma^{\prime}} \psi^{\hbar}(\mathbf{u})^{q}, \quad \forall \mathbf{u} \in \widehat{B}_{4 N_{\hbar}} .
$$

We know from $(*)$ that $N_{\hbar} \rightarrow \infty$ as $\hbar \rightarrow 0$. Arguing exactly as in the proof of Lemma 2.4, we deduce that

$$
\lim _{\hbar \rightarrow 0} R^{\hbar}\left(\boldsymbol{\gamma}, \boldsymbol{\gamma}^{\prime}\right)=R^{0}\left(\gamma, \gamma^{\prime}\right):=\int_{\mathbb{R}^{m}} P_{\gamma, \gamma^{\prime}}\left(\Gamma_{i j}^{0}(\mathbf{u})\right) d \mathbf{u},
$$


and thus

$$
\lim _{\hbar \rightarrow 0} S_{q}^{\hbar}=\bar{S}_{q}^{0}:=\sum_{\substack{\gamma, \gamma^{\prime} \in \mathcal{J}_{m} \\|\gamma|=\left|\gamma^{\prime}\right|=q}} \mathbf{a}^{0}(\gamma) \mathbf{a}^{0}\left(\gamma^{\prime}\right) R^{0}\left(\gamma, \gamma^{\prime}\right)=\int_{\mathbb{R}^{m}} \mathbb{E}\left[\rho_{q}^{0}(0) \rho_{q}^{0}(\mathbf{u})\right] d \mathbf{u} .
$$

Since $S_{q}^{\hbar} \geq 0, \forall q, \hbar$, we have

$$
S_{q}^{\hbar} \geq 0, \quad \forall q
$$

We denote by $\mathcal{P}_{>Q}$ the projection

$$
\mathcal{P}_{>Q}=\sum_{q>Q} \mathcal{P}_{q}
$$

where $\mathcal{P}_{q}$ denotes the projection on the $q$ th chaos component of $\widehat{\mathscr{X}}$.

Lemma 2.6. For any positive integer $Q$, we set

$$
S_{>Q}^{\hbar}:=\mathbb{E}\left[\left|\mathcal{P}_{>Q} \zeta^{\hbar}\left(2 N_{\hbar}\right)\right|^{2}\right]=\sum_{q>Q} S_{q}^{\hbar}
$$

Then

$$
\lim _{Q \rightarrow \infty}\left(\sup _{\hbar} S_{>Q}^{\hbar}\right)=0
$$

the series

$$
\sum_{q \geq 1} \bar{S}_{q}^{0}
$$

is convergent and, if $\bar{S}^{0}$ is its sum, then

$$
\bar{S}^{0}=\lim _{\hbar \rightarrow 0} S^{\hbar}=\lim _{\hbar \rightarrow 0} \sum_{q \geq 1} S_{q}^{\hbar} .
$$

Proof. For $\mathbf{x} \in \mathbb{R}^{m}$, we denote by $\theta_{\mathbf{x}}$ the shift operator associated with the stationary fields $\mathbf{Y}^{\hbar}$, that is,

$$
\theta_{\mathbf{x}} Y^{\hbar}(\bullet)=Y^{\hbar}(\bullet+\mathbf{x}) .
$$

This extends to a unitary map $L^{2}(\Omega) \rightarrow L^{2}(\Omega)$ that commutes with the chaos decomposition of $L^{2}(\Omega)$. Moreover, for any box $B$ and any $\hbar \in\left[0, \hbar_{0}\right]$ we have

$$
\mathbf{Z}^{\hbar}(B+\mathbf{x})=\theta_{\mathbf{x}} \mathbf{Z}^{\hbar}(B) .
$$

If we denote by $\mathcal{L}_{\hbar}$ the set

$$
\mathcal{L}_{\hbar}:=\mathbb{A}^{m} \cap \widehat{B}_{4 N_{\hbar}},
$$


then we deduce

$$
\zeta^{\hbar}\left(2 N_{\hbar}\right)=\left(2 N_{\hbar}\right)^{-m / 2} \sum_{\mathbf{p} \in \mathcal{L}_{\hbar}} \theta_{\mathbf{p}} \zeta^{\hbar}(B), \quad B=\widehat{B}_{1}
$$

We have

$$
\mathcal{P}_{>Q} \zeta^{\hbar}\left(2 N_{\hbar}\right)=\left(2 N_{\hbar}\right)^{-m / 2} \sum_{\mathbf{p} \in \mathcal{L}_{\hbar}} \theta_{\mathbf{p}} \mathcal{P}_{>Q} \zeta^{\hbar}(B)
$$

Using the stationarity of $Y^{\hbar}$ we deduce

$$
S_{>Q}^{\hbar}=\mathbb{E}\left[\left|\mathcal{P}_{>Q} \zeta^{\hbar}\left(2 N_{\hbar}\right)\right|^{2}\right]=\left(2 N_{\hbar}\right)^{-m} \sum_{\mathbf{p} \in \mathcal{L}_{\hbar}} v\left(\mathbf{p}, N_{\hbar}\right) \mathbf{E}\left[\mathcal{P}_{>Q} \zeta(B) \cdot \theta_{\mathbf{p}} \mathcal{P}_{>Q} \zeta^{\hbar}(B)\right]
$$

where $v\left(\mathbf{p}, N_{\hbar}\right)$ denotes the number of points $\mathbf{x} \in \mathcal{L}_{\hbar}$ such that $\mathbf{x}-\mathbf{p} \in \widehat{B}_{2 N_{\hbar}}$. Clearly

$$
v\left(\mathbf{p}, N_{\hbar}\right) \leq\left(2 N_{\hbar}\right)^{m} .
$$

With $K$ denoting the positive constant in (2.22), we deduce from Lemma 2.4 whose applicability is guaranteed by ( $\ddagger)$ that we can choose positive numbers $a, \rho$ such that

$$
\psi^{\hbar}(\mathbf{x}) \leq \rho<\frac{1}{K}, \quad \forall|\mathbf{x}|_{\infty}>a, \forall \hbar \in\left[0, \hbar_{0}\right] .
$$

We split $S_{>Q}^{\hbar}$ into two parts,

$$
S_{>Q}^{\hbar}=S_{>Q, 0}^{\hbar}+S_{>Q, \infty}^{\hbar}
$$

where $S_{>Q, 0}^{\hbar}$ is made up of the terms in (2.29) corresponding to points $\mathbf{p} \in \mathcal{L}_{\hbar}$ such that $|\mathbf{p}|_{\infty}<$ $a+1$, while $S_{>Q, \infty}^{\hbar}$ corresponds to points $\mathbf{p} \in \mathcal{L}_{\hbar}$ such that $|\mathbf{p}|_{\infty} \geq a+1$.

We deduce from (2.30) that for $2 M>a+1$ we have

$$
\left|S_{>Q, 0}^{\hbar}\right| \leq\left(2 N_{\hbar}\right)^{-m}(2 a+2)^{m}\left(2 N_{\hbar}\right)^{m} \mathbb{E}\left[\left|\mathcal{P}_{>Q} \zeta^{\hbar}(B)\right|^{2}\right]
$$

Proposition 2.1(iv) implies that, as $Q \rightarrow \infty$, the right-hand side of the above inequality goes to 0 uniformly with respect to $\hbar$.

To estimate $S_{>Q, \infty}^{\hbar}$ observe that for $\mathbf{p} \in \mathcal{L}_{\hbar}$ such that $|\mathbf{p}|_{\infty}>a+1$ we have

$$
\mathbf{E}\left[\mathcal{P}_{>Q} \zeta^{\hbar}(B) \cdot \theta_{\mathbf{p}} \mathcal{P}_{>Q} \zeta^{\hbar}(B)\right]=\sum_{q>Q} \int_{B} \int_{B} \mathbb{E}\left[\rho_{q}^{\hbar}(\mathbf{x}) \rho_{q}^{\hbar}(\mathbf{y}+\mathbf{p})\right] d \mathbf{x} d \mathbf{y}
$$

where we recall from (2.19) that

$$
\rho_{q}^{\hbar}(\mathbf{x})=\sum_{\gamma \in \mathcal{J}_{m},|\gamma|=q} \mathbf{a}^{\hbar}(\gamma) H_{\gamma}\left(\Xi^{\hbar}(\mathbf{x})\right), \quad \mathcal{J}_{m}:=\mathbb{N}_{0}^{m} \times \mathbb{N}_{0}^{v(m)}, \quad v(m)=\frac{m(m+1)}{2} .
$$


Thus,

$$
\mathbb{E}\left[\rho_{q}^{\hbar}(\mathbf{x}) \rho_{q}^{\hbar}(\mathbf{y}+\mathbf{p})\right]=\mathbb{E}\left[\left(\sum_{\substack{\gamma \in \mathcal{J}_{m},|\gamma|=q}} \mathbf{a}^{\hbar}(\gamma) H_{\gamma}\left(\Xi^{\hbar}(\mathbf{x})\right)\right)\left(\sum_{\substack{\gamma \in \mathcal{J}_{m},|\gamma|=q}} \mathbf{a}^{\hbar}(\gamma) H_{\gamma}\left(\Xi^{\hbar}(\mathbf{y}+\mathbf{p})\right)\right)\right] .
$$

Arcones' inequality [3], Lemma 1, implies that

$$
\mathbb{E}\left[\rho_{q}^{\hbar}(\mathbf{x}) \rho_{q}^{\hbar}(\mathbf{y}+\mathbf{p})\right] \leq K^{q} \psi^{\hbar}(\mathbf{p}+\mathbf{y}-\mathbf{x})^{q} \sum_{\substack{\gamma \in \mathcal{J}_{m},|\gamma|=q}}\left|\mathbf{a}^{\hbar}(\gamma)\right|^{2} \gamma !
$$

We are not out of the woods yet since the series $\sum_{\gamma \in \mathcal{J}_{m}}\left|\mathbf{a}^{\hbar}(\gamma)\right|^{2} \gamma !$ is divergent. Arguing as in $[17,32]$, we deduce that

$$
\sum_{\substack{\gamma \in \mathcal{J}_{m},|\gamma|=q}}\left|\mathbf{a}^{\hbar}(\gamma)\right|^{2} \gamma ! \leq \omega_{\hbar}(2 \pi)^{-\frac{m}{2}} q^{m} \sum_{\substack{\beta \in \mathbb{N}_{0}^{\nu(m)} \\|\beta| \leq q}}\left(f_{\beta}^{\hbar}\right)^{2} \beta ! \leq C q^{m} \mathbb{E}\left[\left|\operatorname{det} \nabla^{2} Y^{\hbar}(0)\right|^{2}\right] .
$$

Using this in (2.31) and (2.32), we deduce

$$
\begin{aligned}
\mathbb{E} & {\left[\mathcal{P}_{>Q} \zeta^{\hbar}(B) \cdot \theta_{\mathbf{p}} \mathcal{P}_{>Q} \zeta^{\hbar}(B)\right] } \\
& \leq \underbrace{C \mathbb{E}\left[\left|\operatorname{det} \nabla Y^{\hbar}(0)\right|^{2}\right]}_{=: C^{\prime}} \sum_{q>Q} q^{m} K^{q} \int_{B} \int_{B} \psi^{\hbar}(\mathbf{s}+\mathbf{u}-\mathbf{t})^{q} d \mathbf{u} d \mathbf{t} .
\end{aligned}
$$

Hence,

$$
\left|S_{>Q, \infty}^{\hbar}\right| \leq C^{\prime}\left(\sum_{q>Q} q^{m} K^{q} \rho^{q-1}\right)\left(\sum_{\substack{\mathbf{p} \in \mathcal{L}_{\hbar},|\mathbf{s}|_{\infty}>a+1}} \int_{B} \int_{B} \psi^{\hbar}(\mathbf{p}+\mathbf{y}-\mathbf{x}) d \mathbf{y} d \mathbf{x}\right)
$$

where we have used the fact that for $|p|_{\infty} \geq a+1,|\mathbf{y}|,|\mathbf{x}| \leq 1$ we have $\psi^{\hbar}(\mathbf{p}+\mathbf{y}-\mathbf{x})<\rho$. Since $\rho<\frac{1}{K}$, the sum

$$
\sum_{q>Q} q^{m} K^{q} \rho^{q-1}
$$

is the tail of a convergent power series. On the other hand,

$$
\begin{aligned}
\sum_{\substack{\mathbf{p} \in \mathcal{L}_{\hbar},|\mathbf{p}|_{\infty}>a+1}} \int_{B} \int_{B} \psi^{\hbar}(\mathbf{p}+\mathbf{y}-\mathbf{x}) d \mathbf{y} d \mathbf{x} & \leq \sum_{\mathbf{p} \in \mathcal{L}_{\hbar}} \int_{[-1,1]^{m}} \psi^{\hbar}(\mathbf{p}+\mathbf{y}) \\
& \leq 2 \int_{\mathbb{R}^{m}} \psi^{\hbar}(\mathbf{y}) d \mathbf{y} \stackrel{(2.16)}{=} O(1) .
\end{aligned}
$$


This proves that $\sup _{\hbar}\left|S_{>Q, \infty}^{\hbar}\right|$ goes to zero as $Q \rightarrow \infty$ and completes the proof of (2.25). The claim (2.26) follows immediately from (2.25). This concludes the proof of Lemma 2.6 and of the fact $\mathbf{F}_{1}$.

Proof of $\mathbf{F}_{2}$. In [32], we have shown that the limit $S^{0}$ in (2.17) is the sum of a series

$$
S^{0}=\sum_{q \geq 1} S_{q}^{0}, \quad S_{q}^{0}=\int_{\mathbb{R}^{m}} \mathbb{E}\left[\rho_{q}^{0}(0) \rho_{q}^{0}(\mathbf{u})\right] d \mathbf{u} .
$$

The equality (2.24) shows that $\bar{S}^{0}=S^{0}>0$. This concludes the proof of Proposition 2.5.

\subsection{Proof of Theorem 1.2}

In [32], we have shown that, as $\hbar \rightarrow 0$, the random variables converge in law to a random variable $\sim \mathcal{N}\left(0, S^{0}\right)$.

As explained in Section 1.5, to conclude the proof of Theorem 1.2 it suffices to establish the asymptotic normality as $\hbar \rightarrow 0$ of the family

$$
\zeta_{q}^{\hbar}=\frac{1}{\left(2 N_{\hbar}\right)^{m / 2}} \int_{\widehat{B}_{2 N_{\hbar}}} \rho_{q}(\mathbf{x}) d \mathbf{x}, \quad \forall q \geq 1 .
$$

This follows from the fourth-moment theorem [34], Theorem 5.2.7, [36]. Here are the details.

Recall from [22], IV.1, that we have a surjective isometry $\Theta_{q}: \mathscr{X}^{\odot q} \rightarrow \mathscr{X}: q$ : where $\mathscr{X} \odot q$ is the $q$ th symmetric power and $\mathscr{X}^{:} q:$ is the $q$ th chaos component of $\widehat{\mathscr{X}}$. The multiple Itô integral $\mathbf{I}_{q}$ is then the map

$$
\mathbf{I}_{q}=\frac{1}{\sqrt{q !}} \Theta_{q} .
$$

We can write $\zeta_{q}^{\hbar}$ as a multiple Itô integral

$$
\zeta_{q}^{\hbar}=\mathbf{I}_{q}\left[g_{q}^{\hbar}\right], \quad g_{q}^{\hbar} \in \mathscr{X}^{\odot q} .
$$

According to [34], Theorem 5.2.7(v), to prove that $\zeta_{q}^{\hbar}$ converge in law to a normal variable it suffices to show that

$$
\lim _{\hbar \rightarrow 0}\left\|g_{q}^{\hbar} \otimes_{r} g_{q}^{\hbar}\right\|_{\mathscr{X} \otimes(2 q-2 r)}=0, \quad \forall r=1, \ldots, q-1 .
$$

In our context, using the isometry $\mathbf{I}$ in (1.14) we can view $g_{q}^{\hbar}$ as a function

$$
g_{q}^{\hbar} \in L^{2}\left(\left(\mathbb{R}^{m} \times \mathbb{R}^{m}\right)^{q}\right), \quad g_{q}^{\hbar}=g_{q}^{\hbar}\left(\mathbf{z}_{1}, \ldots, \mathbf{z}_{q}\right), \quad \mathbf{z}_{j} \in \mathbb{R}^{m} \times \mathbb{R}^{m},
$$


and then

$$
\begin{aligned}
& g_{q}^{\hbar} \otimes_{r} g_{q}^{\hbar} \in L^{2}\left(\left(\mathbb{R}^{m} \times \mathbb{R}^{m}\right)^{2(q-r)}\right), \\
& g_{q}^{\hbar} \otimes_{r} g_{q}^{\hbar}\left(\mathbf{z}_{q-r+1}, \mathbf{z}_{q-r+1}^{\prime}, \ldots, \mathbf{z}_{q}, \mathbf{z}_{q}^{\prime}\right) \\
& \quad=\int_{\left(\mathbb{R}^{m} \times \mathbb{R}^{m}\right)^{r}} g_{q}^{\hbar}\left(\mathbf{z}_{1}, \ldots, \mathbf{z}_{q}, \mathbf{z}_{q-r+1}, \ldots, \mathbf{z}_{q}\right) g_{q}^{\hbar}\left(\mathbf{z}_{1}, \ldots, \mathbf{z}_{q}, \mathbf{z}_{q-r+1}^{\prime}, \ldots, \mathbf{z}_{q}^{\prime}\right) d \mathbf{z}_{1} \cdots d \mathbf{z}_{q} .
\end{aligned}
$$

To show (2.33) we invoke the arguments following the inequality (18) in the second step of the proof of [17], Proposition 2.4, which extend with no modification to the setup in this paper.

\subsection{Proof of Theorem 1.3}

We set

$$
N_{\hbar}:=\left\lfloor\frac{r}{2 \hbar}\right\rfloor, \quad s_{\hbar}:=\frac{r}{2 \hbar N_{\hbar}} .
$$

Then $N_{\hbar} \in \mathbb{N}$,

$$
\lim _{\hbar \rightarrow 0} N_{\hbar}=\infty, \quad \widehat{B}_{2 N_{\hbar} s_{\hbar}}=\widehat{B}_{\hbar^{-1} r} \subset \widehat{B}_{1 /(2 \hbar)}, \quad \lim _{\hbar \rightarrow 0} s_{\hbar}=1 .
$$

Thus, $\widehat{B}_{\hbar^{-1} r}$ is a cube, centered at 0 with vertices in the lattice $\left(s_{\hbar} \mathbb{Z}\right)^{m}$ and $s(\hbar) \approx 1$ for $\hbar$ small.

To reach the conclusion, (i) run the arguments in the proof of Theorem 1.1 with the following modified notations: the box $\widehat{B}_{2 N_{\hbar}}$ should be replaced with the box $\widehat{B}_{2 N_{\hbar} s_{\hbar}}=s_{\hbar} \widehat{B}_{2 N_{\hbar}}$, the lattice $\mathbb{A}^{m}$ in (2.13) replaced by $s_{\hbar} \mathbb{A}^{m}$, and $\widehat{B}(\mathbf{a})$ redefined as $s_{\hbar} \widehat{B}_{1}(\mathbf{a})=\widehat{B}_{s_{\hbar}}(\mathbf{a})$.

To reach the conclusions (ii) and (iii) of Theorem 1.3, run the arguments in the Sections 2.4 and 2.5 with the following modified notations: the box $\widehat{B}_{2 N_{\hbar}}$ should be replaced with the box $\widehat{B}_{2 N_{\hbar} s_{\hbar}}=s_{\hbar} \widehat{B}_{2 N_{\hbar}}$, in (2.27) the set $\mathcal{L}_{\hbar}$ should be redefined to be

$$
\mathcal{L}_{\hbar}:=s_{\hbar}\left(\mathbb{A}^{m} \cap \widehat{B}_{4 N_{\hbar}}\right),
$$

and the box $B$ in (2.28) should be redefined to be $\widehat{B}_{s_{\hbar}}=s_{\hbar} \widehat{B}_{1}$.

Remark 2.7. The above proof shows that for any $r \in(0,1 / 2]$, we have

$$
\lim _{Q \rightarrow \infty}\left(\sup _{\hbar} \mathbb{E}\left[\left|\mathcal{P}_{>Q} \zeta^{\hbar}(r / \hbar)\right|^{2}\right]\right)=0
$$

\subsection{Proof of Theorem 1.5}

Note that (see (1.17) for notation)

$$
\mathbf{Z}\left(X^{\hbar}, \mathbb{T}^{m}\right)=\mathbf{Z}^{\hbar}(1 / h)
$$

Hence, it suffices to prove: 
(i) As $\hbar \rightarrow 0$

$$
\mathbf{Z}^{\hbar}(1 / h)=\hbar^{-m}\left(\bar{Z}_{0}+O\left(\hbar^{\infty}\right)\right)
$$

(ii) As $\hbar \rightarrow 0$

$$
\operatorname{var}\left[\mathbf{Z}^{\hbar}(1 / h)\right] \sim S^{0} \cdot(1 / \hbar)^{m} .
$$

(iii) The families of random variables

$$
\left\{\zeta^{\hbar}(1 / \hbar)\right\}_{\hbar \in\left(0, \hbar_{0}\right]} \text { and }\left\{\zeta^{0}(1 / \hbar)\right\}_{\hbar \in\left(0, \hbar_{0}\right]}
$$

converge in distribution as $\hbar \rightarrow 0$ to normal random variables $\sim \mathcal{N}\left(0, S^{0}\right)$.

From this point of view, these results extend Theorem 1.3 to the case $r=1$. We cannot invoke Theorem 1.3 (or Theorem 1.2) with $2 N_{\hbar}$ formally replaced by $\frac{1}{\hbar}$ to prove (ii) and (iii) because the condition ( $\$)$ is violated. To prove these facts, we will deploy a new technique, that takes advantage of the fact that $B_{1}$ is a fundamental domain of the lattice $\mathbb{Z}^{m}$.

Let us first observe that it suffices to show that the variables $\zeta^{\hbar}(1 / \hbar)$ satisfy the conclusions of Proposition 2.5. Once we verify this fact, the arguments in Section 2.5 extend without any modification to this case yielding Theorem 1.5.

Let us first show $\mathbf{F}_{1}$, that is, the limit

$$
\bar{S}^{0}:=\lim _{h \searrow 0} \operatorname{var}\left[\zeta^{\hbar}(1 / h)\right]
$$

exists and it is finite. Using (2.20) (with $2 N_{\hbar}$ replaced by $1 / \hbar$ ), we deduce

$$
S_{q}^{\hbar}=\mathcal{P}_{q} \zeta(1 / \hbar)=\sum_{\substack{\gamma, \gamma^{\prime} \in \mathcal{J}_{m} \\|\gamma|=\left|\gamma^{\prime}\right|=q}} \mathbf{a}^{\hbar}(\gamma) \mathbf{a}^{\hbar}\left(\gamma^{\prime}\right) \int_{\widehat{B}_{2 / \hbar}} P_{\gamma, \gamma^{\prime}}\left(\Gamma_{i j}^{\hbar}(\mathbf{u})\right) \prod_{k=1}^{m}\left(1-\hbar\left|u_{k}\right|\right) d \mathbf{u}
$$

We set

$$
\boldsymbol{\Omega}_{m}:=\{ \pm 1\}^{m} \subset \mathbb{R}^{m} .
$$

For $\boldsymbol{\omega} \in \boldsymbol{\Omega}_{m}$ we denote by $\mathcal{O}^{\boldsymbol{\omega}}$ the "octant" in $\mathbb{R}^{m}$ that contains $\boldsymbol{\omega}$, that is,

$$
\mathcal{O}^{\omega}:=\left\{\mathbf{x} \in \mathbb{R}^{m} ; x_{i} \omega_{i}>0, \forall i\right\}, \quad \widehat{B}_{R}^{\omega}:=\widehat{B}_{R} \cap \mathcal{O}^{\omega} .
$$

Note that

$$
\begin{aligned}
& \int_{\widehat{B}_{2 / \hbar}} P_{\gamma, \gamma^{\prime}}\left(\Gamma_{i j}^{\hbar}(\mathbf{u})\right) \prod_{k=1}^{m}\left(1-\hbar\left|u_{k}\right|\right) d \mathbf{u} \\
& =\sum_{\boldsymbol{\omega} \in \boldsymbol{\Omega}_{m}} \int_{\widehat{B}_{2 / \hbar}^{\omega}} P_{\gamma, \gamma^{\prime}}\left(\Gamma_{i j}^{\hbar}(\mathbf{u})\right) \prod_{k=1}^{m}\left(1-\hbar\left|u_{k}\right|\right) d \mathbf{u} .
\end{aligned}
$$





Figure 1. The decomposition (2.37) in the case $m=2, \hbar=1, \omega=(1,1)$. The $2^{m}$ quadrants decompose $\widehat{B}_{1}$ into $2^{m}$ half-sized squares that can be reassembled back into $\widehat{B}_{2}^{\omega}$ via the $2^{m}$ translations by vectors in $\left(\hbar^{-1} \mathbb{Z}\right)^{m}$ that map the $2^{m}$ vertices of $\widehat{B}_{1}$ to the center of $\widehat{B}_{2}^{\omega}$.

For any $\boldsymbol{\omega} \in \boldsymbol{\Omega}_{m}$ the cube $\widehat{B}_{2 / \hbar}^{\boldsymbol{\omega}}$ it is centered at $\frac{1}{2 \hbar} \boldsymbol{\omega}$ and it has size $\frac{1}{\hbar}$. For any $\boldsymbol{v} \in \boldsymbol{\Omega}_{m}$ the cube $\widehat{B}_{1 / \hbar}$ has a unique vertex in $\mathcal{O}^{v}$, namely $\frac{1}{2 \hbar} \boldsymbol{v}$. We obtain a decomposition (see Figure 1)

$$
\widehat{B}_{2 / \hbar}^{\omega} \backslash H=\bigcup_{\boldsymbol{v} \in \boldsymbol{\Omega}_{m}}\left(\frac{1}{2 \hbar}(\boldsymbol{\omega}-\boldsymbol{v})+\widehat{B}_{1 / \hbar}^{\boldsymbol{v}}\right), \quad H=\bigcup_{i=1}^{m}\left\{x_{i}=0\right\} .
$$

Denote by $L_{\hbar}$ the lattice $\left(\hbar^{-1} \mathbb{Z}\right)^{m}$. Note that the integrand $P_{\gamma, \gamma^{\prime}}\left(\Gamma_{i j}^{\hbar}(\mathbf{u})\right)$ is $L_{\hbar}$-periodic because $V^{\hbar}$ is such. The "miraculous" quantization condition

$$
\frac{1}{2 \hbar}(\boldsymbol{\omega}-\boldsymbol{v}) \in L_{\hbar}, \quad \forall \boldsymbol{\omega}, \boldsymbol{v} \in \boldsymbol{\Omega}_{m}
$$


allows us to simplify dramatically the right-hand side of (2.37). More precisely, we have

$$
\begin{aligned}
& \int_{\widehat{B}_{2 / \hbar}^{\omega}} P_{\gamma, \gamma^{\prime}}\left(\Gamma_{i j}^{\hbar}(\mathbf{u})\right) \prod_{k=1}^{m}\left(1-\hbar\left|u_{k}\right|\right) d \mathbf{u} \\
& =\sum_{\mathbf{v} \in \mathbf{\Omega}_{m}} \int_{\widehat{B}_{1 / \hbar}^{v}} P_{\gamma, \gamma^{\prime}}\left(\Gamma_{i j}^{\hbar}(\mathbf{v})\right) \prod_{k=1}^{m}\left(1-\hbar\left|v_{k}+\frac{1}{2 \hbar}\left(\omega_{k}-v_{k}\right)\right|\right) d \mathbf{v} \\
& =\int_{\widehat{B}_{1 / \hbar}} P_{\gamma, \gamma^{\prime}}\left(\Gamma_{i j}^{\hbar}(\mathbf{v})\right) f_{\omega}^{\hbar}(\mathbf{v}) d \mathbf{v},
\end{aligned}
$$

where

$$
\left.f_{\boldsymbol{\omega}}^{\hbar}(\mathbf{v})\right|_{\mathcal{O}^{v}}:=\prod_{k=1}^{m}\left(1-\hbar\left|v_{k}+\frac{1}{2 \hbar}\left(\omega_{k}-v_{k}\right)\right|\right), \quad \forall \boldsymbol{v} \in \boldsymbol{\Omega}_{m},
$$

and $f_{\boldsymbol{\omega}}^{\hbar}(\mathbf{v})=0$ if $\mathbf{v}$ lies on one of the coordinate hyperplanes $\left\{x_{i}=0\right\} \subset \mathbb{R}^{m}$. Hence,

$$
\int_{\widehat{B}_{2 / \hbar}} P_{\gamma, \gamma^{\prime}}\left(\Gamma_{i j}^{\hbar}(\mathbf{u})\right) \prod_{k=1}^{m}\left(1-\hbar\left|u_{k}\right|\right) d \mathbf{u}=\int_{\widehat{B}_{1 / \hbar}} P_{\gamma, \gamma^{\prime}}\left(\Gamma_{i j}^{\hbar}(\mathbf{v})\right)\left(\sum_{\omega \in \mathbf{\Omega}_{m}} f_{\omega}^{\hbar}(\mathbf{v})\right) d \mathbf{v} .
$$

Now observe that

$$
\begin{aligned}
\left|f_{\omega}^{\hbar}(\mathbf{v})\right| \leq 3^{m}, \quad \forall \boldsymbol{\omega}, \forall \mathbf{v} \in \mathbb{R}^{m} & \\
\lim _{\hbar \searrow 0} f_{\omega}^{\hbar}(\mathbf{v}) & =f_{0}^{\omega}(\mathbf{v})=\mathbf{1}_{\mathcal{O}^{\omega}} .
\end{aligned}
$$

Using (2.39), (2.40a), (2.40b) and (2.22), we deduce exactly as in the proof of Lemma 2.4 that

$$
\lim _{\hbar \searrow 0} \int_{\widehat{B}_{2 / \hbar}} P_{\gamma, \gamma^{\prime}}\left(\Gamma_{i j}^{\hbar}(\mathbf{u})\right) \prod_{k=1}^{m}\left(1-\hbar\left|u_{k}\right|\right) d \mathbf{u}=R^{0}\left(\gamma, \gamma^{\prime}\right):=\int_{\mathbb{R}^{m}} P_{\gamma, \gamma^{\prime}}\left(\Gamma_{i j}^{0}(\mathbf{u})\right) d \mathbf{u} .
$$

Hence,

$$
\lim _{\hbar \rightarrow 0} S_{q}^{\hbar}=\bar{S}_{q}^{0}:=\sum_{\substack{\gamma, \gamma^{\prime} \in \mathcal{J}_{m} \\|\gamma|=\left|\gamma^{\prime}\right|=q}} \mathbf{a}^{0}(\gamma) \mathbf{a}^{0}\left(\gamma^{\prime}\right) R^{0}\left(\gamma, \gamma^{\prime}\right)=\int_{\mathbb{R}^{m}} \mathbb{E}\left[\rho_{q}^{0}(0) \rho_{q}^{0}(\mathbf{u})\right] d \mathbf{u} .
$$

This is the counterpart of (2.24).

Let us explain how to prove Lemma 2.6 in this context when ( $\ddagger)$ is not satisfied. We set

$$
\boldsymbol{\Omega}_{m}^{\hbar}=\frac{1}{4 \hbar} \cdot \boldsymbol{\Omega}_{m}
$$

The point $\omega^{\hbar}:=(4 \hbar)^{-1} \boldsymbol{\omega}$ in $\boldsymbol{\Omega}_{m}^{\hbar}$ is the center of the cube $\widehat{B}_{1 / \hbar}^{\omega}=\widehat{B}_{1 / \hbar} \cap \mathcal{O}^{\omega}$. We have

$$
\zeta(1 / \hbar)=(\hbar)^{m / 2}\left(\mathbf{Z}^{\hbar}\left(\widehat{B}_{1 / h}\right)-\mathbb{E}\left[\mathbf{Z}^{\hbar}\left(\widehat{B}_{1 / \hbar}\right)\right]\right) .
$$


From the equality

$$
\widehat{B}_{1 / \hbar}=\bigcup_{\omega^{\hbar} \in \mathbf{\Omega}_{m}^{\hbar}} \widehat{B}_{1 / 2 \hbar}\left(\omega^{\hbar}\right)
$$

we deduce

$$
\mathbf{Z}^{\hbar}\left(\widehat{B}_{1 / h}\right)-\mathbb{E}\left[\mathbf{Z}^{\hbar}\left(\widehat{B}_{1 / \hbar}\right)\right]=\sum_{\omega^{\hbar} \in \mathbf{\Omega}_{m}^{\hbar}} \theta_{\omega^{\hbar}} \mathbf{Z}^{\hbar}\left(\widehat{B}_{1 /(2 \hbar)}\right)-\mathbb{E}\left[\mathbf{Z}^{\hbar}\left(\widehat{B}_{1 /(2 \hbar)}\right)\right]
$$

Since

$$
\left|\widehat{B}_{1 /(2 \hbar)}\right|=(2 \hbar)^{-m},
$$

we deduce

$$
\zeta(1 / \hbar)=\frac{1}{2^{m / 2}} \sum_{\boldsymbol{\omega}^{\hbar} \in \mathbf{\Omega}_{m}^{\hbar}} \theta_{\boldsymbol{\omega}^{\hbar}} \zeta\left(\widehat{B}_{1 /(2 \hbar)}\right)
$$

In particular,

$$
\mathcal{P}_{>Q} \zeta(1 / \hbar)=\frac{1}{2^{m / 2}} \sum_{\omega^{\hbar} \in \boldsymbol{\Omega}_{m}^{\hbar}} \theta_{\boldsymbol{\omega}^{\hbar}} \mathcal{P}_{>Q} \zeta\left(\widehat{B}_{1 /(2 \hbar)}\right)
$$

Hence,

$$
\begin{aligned}
\mathbb{E}\left[\left|\mathcal{P}_{>Q} \zeta(1 / \hbar)\right|^{2}\right] & =\frac{1}{2^{m}} \sum_{\boldsymbol{\omega}^{\hbar}, \boldsymbol{v}^{\hbar} \in \boldsymbol{\Omega}_{m}^{\hbar}} \mathbb{E}\left[\theta_{\boldsymbol{\omega}^{\hbar}} \mathcal{P}_{>Q} \zeta\left(\widehat{B}_{1 /(2 \hbar)}\right) \cdot \theta_{\boldsymbol{\omega}^{\hbar}} \mathcal{P}_{>Q} \zeta\left(\widehat{B}_{1 /(2 \hbar)}\right)\right] \\
& \leq \frac{1}{2^{m}} \sum_{\boldsymbol{\omega}^{\hbar}, \boldsymbol{v}^{\hbar} \in \boldsymbol{\Omega}_{m}^{\hbar}} \mathbb{E}\left[\left|\mathcal{P}_{>Q} \zeta\left(\widehat{B}_{1 /(2 \hbar)}\right)\right|^{2}\right] \\
& =2^{m} \mathbb{E}\left[\left|\mathcal{P}_{>Q} \zeta\left(\widehat{B}_{1 /(2 \hbar)}\right)\right|^{2}\right]
\end{aligned}
$$

Using (2.34), we deduce

$$
\lim _{Q \rightarrow \infty}\left(\sup _{\hbar} \mathbb{E}\left[\left|\mathcal{P}_{>Q} \zeta^{\hbar}\left(\widehat{B}_{1 /(2 \hbar)}\right)\right|^{2}\right]\right)=0
$$

showing that

$$
\lim _{Q \rightarrow \infty} \sup _{\hbar} \mathbb{E}\left[\left|\mathcal{P}_{>Q} \zeta(1 / \hbar)\right|^{2}\right]=0 .
$$

This shows that the variables $\zeta^{\hbar}(1 / \hbar)$ satisfy the conclusions of Lemma 2.6.

This shows that the $\operatorname{limit} \lim _{h \searrow 0} \operatorname{var}\left[\zeta^{\hbar}(1 / h)\right]$ exists and it is finite. Moreover, (2.42) shows that this limit is $S_{0}$. 


\section{Appendix: Proof of Proposition 2.1}

We will follow the strategy in the proof of [17], Proposition 1.1. Several modifications are required since the random functions $Y^{\hbar}$ are not isotropic for $\hbar>0$.

Denote by $p_{\mathbf{x}}^{\hbar}(-)$ and $p_{\mathbf{x}, \mathbf{y}}^{\hbar}(-,-)$ the probability densities of the Gaussian vectors $\nabla Y^{\hbar}(\mathbf{x})$ and respectively, $\nabla Y^{\hbar}(\mathbf{x}) \oplus \nabla Y^{\hbar}(\mathbf{y})$. For simplicity, we denote by $|S|$ the Lebesgue volume of a Borel subset $S \subset \mathbb{R}^{m}$. Due to the stationarity of $Y^{\hbar}$, it suffices to assume that the box $B$ is centered at 0 .

Let us observe that the covariance matrix of the stationary Gaussian random vector $\nabla Y^{\hbar}(\mathbf{x})$ is $-\nabla^{2} V^{\hbar}(0)$ and

$$
\left\|\nabla^{2} V^{\hbar}(0)-\nabla^{2} V(0)\right\|=O\left(\hbar^{\infty}\right) .
$$

On the other hand, $\nabla^{2} V(0)$ is a nonzero multiple of the identity matrix. Thus, for all $\hbar$ sufficiently small the Gaussian random vector $\nabla Y^{\hbar}(\mathbf{x})$ is nondegenerate for any $\mathbf{x}$.

As explained in [31], Section 3.2, if $\hbar$ is sufficiently small, the Gaussian random vector

$$
\nabla Y^{\hbar}(\mathbf{x}) \oplus \nabla Y^{\hbar}(\mathbf{y})
$$

is nondegenerate for any $\mathbf{x} \neq \mathbf{y} \bmod \left(\hbar^{-1} \mathbb{Z}\right)^{m}$. have

Using the Kac-Rice formula [2], Chapter 11, or [11], Chapter 6, we deduce that, $\forall \mathbf{v} \in \mathbb{R}^{m}$ we

$$
\begin{aligned}
& \mathbb{E}\left[\mathbf{Z}^{\hbar}(\mathbf{v}, B)\right]=\mathbb{E}\left[\left|\operatorname{det} \nabla^{2} Y^{\hbar}(0)\right|\right] p_{0}^{\hbar}(\mathbf{v})|B|, \\
& \mathbb{E}\left[\mathbf{Z}^{\hbar}(\mathbf{v}, B)\left(\mathbf{Z}^{\hbar}(\mathbf{v}, B)-1\right)\right] \\
& \quad=\int_{2 B}|B \cap(B-\mathbf{y})| \underbrace{\mathbb{E}_{\mathbf{y}, \mathbf{v}}\left[\left|\operatorname{det} \nabla^{2} Y^{\hbar}(0) \operatorname{det} \nabla^{2} Y^{\hbar}(\mathbf{y})\right|\right]}_{g^{\hbar}(\mathbf{v}, \mathbf{y})} p_{0, \mathbf{y}}(\mathbf{v}, \mathbf{v}) d \mathbf{y},
\end{aligned}
$$

where, for typographical reasons, we denoted by $\mathbb{E}_{\mathbf{y}, \mathbf{v}}$ the conditional expectation

$$
\mathbb{E}_{\mathbf{y}, \mathbf{v}}[-]=\mathbb{E}\left[-\mid C_{\mathbf{y}}(\mathbf{v})\right], \quad C_{\mathbf{y}}(\mathbf{v}):=\left\{\nabla Y^{\hbar}(0)=\nabla Y^{\hbar}(\mathbf{y})=\mathbf{v}\right\}
$$

The two sides of the equality (A.2) are simultaneously finite or infinite.

Let us point out that the integrand on the right-hand side of this equality could blow-up at $\mathbf{y}=0$ because the Gaussian vector $\nabla Y^{\hbar}(0) \oplus \nabla Y^{\hbar}(0)$ is degenerate and therefore

$$
\lim _{\mathbf{y} \rightarrow 0} p_{0, \mathbf{y}}^{\hbar}(\mathbf{v}, \mathbf{v})=\infty
$$

The most demanding part in the proof of Proposition 2.1 is showing that the right-hand side of (A.2) is finite. This boils down to understanding the singularity at the origin of the integrand in (A.2). In [31], we proved this fact in the case $\mathbf{v}=0$. To deal with the general case, we will use a blend of the ideas in [17] and [31]. 
Step 1 . We will show that there exist $\hbar_{1}>0, r_{1}>0$ and $C_{1}>0$ such that for any $\hbar \leq h_{1}$ we have

$$
\begin{aligned}
p_{0, \mathbf{y}}^{\hbar}(0,0) & <\infty, \quad \forall \mathbf{y} \neq 0, \\
0 & <p_{0, \mathbf{y}}^{\hbar}(\mathbf{v}, \mathbf{v}) \leq C_{1}|\mathbf{y}|^{-m}, \quad \forall 0<|\mathbf{y}|<r_{1}, \forall \mathbf{v} \in \mathbb{R}^{m} .
\end{aligned}
$$

These two facts follow from [31], Lemma 3.5, and the obvious inequality

$$
p_{0, \mathbf{y}}^{\hbar}(\mathbf{v}, \mathbf{v}) \leq p_{0, \mathbf{y}}^{\hbar}(0,0) .
$$

Step 2. We will show that there exist $\hbar_{2}>0, r_{2}>0$ and $C_{2}>0$ such that, for any $\hbar \leq h_{2}$, we have

$$
\left|g^{\hbar}(\mathbf{v}, \mathbf{y})\right| \leq C_{2}\|\mathbf{y}\|^{2}, \quad \forall|\mathbf{y}| \leq r_{2}, \mathbf{v} \in \mathbb{R}^{m}
$$

We set

$$
f^{\hbar}(\mathbf{y}, \mathbf{v}):=\mathbf{E}_{\mathbf{y}, \mathbf{v}}\left[\left|\operatorname{det} \nabla^{2} Y^{\hbar}(0)\right|^{2}\right]
$$

From the Cauchy inequality and the stationarity of $Y^{\hbar}$, we deduce

$$
\begin{aligned}
g^{\hbar}(\mathbf{v}, \mathbf{y})^{2} & \leq \mathbf{E}_{\mathbf{y}, \mathbf{v}}\left[\left|\operatorname{det} \nabla^{2} Y^{\hbar}(0)\right|^{2}\right] \cdot \mathbf{E}_{\mathbf{y}, \mathbf{v}}\left[\left|\operatorname{det} \nabla^{2} Y^{\hbar}(\mathbf{y})\right|^{2}\right] \\
& =f^{\hbar}(\mathbf{y}, \mathbf{v}) f^{\hbar}(-\mathbf{y}, \mathbf{v})=f^{\hbar}(\mathbf{y}, \mathbf{v})^{2} .
\end{aligned}
$$

We now invoke Hadamard's inequality [21], Theorem 7.8.1: if $A: \mathbb{R}^{m} \rightarrow \mathbb{R}^{m}$ is an $m \times m$ symmetric positive operator and $\left\{\mathbf{e}_{1}, \ldots, \mathbf{e}_{m}\right\}$ is an orthonormal basis of $\mathbb{R}^{m}$, then

$$
\operatorname{det} A \leq \prod_{j=1}^{m}\left(A \mathbf{e}_{j}, \mathbf{e}_{j}\right)
$$

Applying this inequality to $A=\nabla^{2} Y^{h}(0)^{2}$ and a fixed orthonormal basis $\left\{\mathbf{e}_{1}, \ldots, \mathbf{e}_{m}\right\}$ such that

$$
\mathbf{e}_{1}:=|\mathbf{y}|^{-1} \mathbf{y}
$$

we deduce

$$
\left|\operatorname{det} \nabla^{2} Y^{\hbar}(0)\right|^{2} \leq|\mathbf{y}|^{-2}\left\|\nabla^{2} Y^{\hbar}(0) \mathbf{y}\right\|^{2}\left\|\nabla^{2} Y^{\hbar}(0)\right\|^{2(m-1)}
$$

Hence,

$$
\begin{aligned}
\left|g^{\hbar}(\mathbf{v}, \mathbf{y})\right| & \leq f^{\hbar}(\mathbf{v}, \mathbf{y}) \mathbb{E}_{\mathbf{y}, \mathbf{v}}\left[\left|\operatorname{det} \nabla^{2} Y^{\hbar}(0)\right|^{2}\right]^{2} \\
& \leq|\mathbf{y}|^{-2} \mathbb{E}_{\mathbf{y}, \mathbf{v}}\left[\left\|\nabla^{2} Y^{y}(0) \mathbf{y}\right\|^{4}\right]^{\frac{1}{2}} \mathbb{E}_{\mathbf{y}, \mathbf{v}}\left[\left\|\nabla^{2} Y^{y}(0)\right\|^{4(m-1)}\right]^{\frac{1}{2}}
\end{aligned}
$$

Now observe that

$$
\left\|\nabla^{2} Y^{\hbar}(0) \mathbf{y}\right\|^{2}=|\mathbf{y}|^{2} \sum_{j=1}^{m} Y_{1 j}^{\hbar}(0)^{2},
$$


where, for any smooth function $F: \mathbb{R}^{m} \rightarrow \mathbb{R}$, we set

$$
F_{i, j, \ldots k}:=\partial_{\mathbf{e}_{i}} \partial_{\mathbf{e}_{j}} \cdots \partial_{\mathbf{e}_{k}} F
$$

Thus,

$$
\begin{aligned}
\left|\nabla^{2} Y^{\hbar}(0) \mathbf{y}\right|^{4} & \leq m|\mathbf{y}|^{4} \sum_{j=1}^{m} Y_{1 j}^{\hbar}(0)^{4} \\
\mathbb{E}_{\mathbf{y}, \mathbf{v}}\left[\left|\nabla^{2} Y^{\hbar}(0) \mathbf{y}\right|^{4}\right] & \leq m|\mathbf{y}|^{4} \sum_{j=1}^{m} \mathbb{E}_{\mathbf{y}, \mathbf{v}}\left[Y_{1 j}^{\hbar}(0)^{4}\right] \\
g^{\hbar}(\mathbf{v}, \mathbf{y}) & \leq \sqrt{m}\left(\sum_{j=1}^{m} \mathbb{E}_{\mathbf{y}, \mathbf{v}}\left[Y_{1 j}^{\hbar}(0)^{4}\right]\right)^{\frac{1}{2}} \mathbb{E}_{\mathbf{y}, \mathbf{v}}\left[\left\|\nabla^{2} Y^{\hbar}(0)\right\|^{4(m-1)}\right]^{\frac{1}{2}} .
\end{aligned}
$$

For each $j=1, \ldots, m$ define the random function

$$
F_{j}:[0,1] \rightarrow \mathbb{R}, \quad F_{j}(t)=Y_{j}^{\hbar}(t \mathbf{y})
$$

Then

$$
F_{j}^{\prime}(t)=|\mathbf{y}| Y_{1, j}^{\hbar}(t \mathbf{y}), \quad F_{j}^{\prime \prime}(t)=|\mathbf{y}|^{2} Y_{1,1, j}^{\hbar}(t \mathbf{y}) .
$$

Using the Taylor formula with integral remainder, we deduce

$$
F_{j}(1)-F_{j}(0)=F_{j}^{\prime}(0)+\int_{0}^{1} F_{j}^{\prime \prime}(t)(1-t) d t,
$$

that is,

$$
Y_{j}^{\hbar}(\mathbf{y})-Y_{j}^{\hbar}(0)=Y_{1 j}^{\hbar}(0)+|\mathbf{y}| \int_{0}^{1} Y_{11 j}^{\hbar}(t \mathbf{y})(t \mathbf{y}) d t
$$

Hence,

$$
Y_{1, j}^{\hbar}(0)=Y_{j}^{\hbar}(\mathbf{y})-Y_{j}^{\hbar}(0)-|\mathbf{y}| \int_{0}^{1} Y_{1,1, j}^{\hbar}(t \mathbf{y})(t \mathbf{y}) d t .
$$

Setting $v_{j}:=\left(\mathbf{v}, \mathbf{e}_{j}\right)$ and observing that under the condition $C_{\mathbf{y}}(\mathbf{v})$, we have

$$
Y_{j}^{\hbar}(0)=Y_{j}^{\hbar}(\mathbf{y})=v_{j}, \quad \forall j=1, \ldots, m,
$$

we deduce

$$
\begin{aligned}
\mathbb{E}_{\mathbf{y}, \mathbf{v}}\left[Y_{1, j}^{\hbar}(0)^{4}\right] & =\mathbb{E}_{\mathbf{y}, \mathbf{v}}\left[\left(Y_{j}^{\hbar}(\mathbf{y})-Y_{j}^{\hbar}(0)-|\mathbf{y}| \int_{0}^{1} Y_{1,1, j}^{\hbar}(t \mathbf{y})(1-t) d t\right)^{4} \mid C_{\mathbf{y}}(\mathbf{v})\right] \\
& =|\mathbf{y}|^{4} \mathbb{E}_{\mathbf{y}, \mathbf{v}}\left[\left(\int_{0}^{1} Y_{1,1, j}^{\hbar}(t \mathbf{y})(1-t) d t\right)^{4}\right] \leq|\mathbf{y}|^{4} \mathbb{E}_{\mathbf{y}, \mathbf{v}}\left[\int_{0}^{1}\left|Y_{1,1, j}^{\hbar}(t \mathbf{y})\right|^{4} d t\right]
\end{aligned}
$$




$$
=|\mathbf{y}|^{4} \int_{0}^{1} \mathbb{E}_{\mathbf{y}, \mathbf{v}}\left[\left|Y_{1,1, j}^{\hbar}(t \mathbf{y})\right|^{4}\right] d t
$$

We conclude that

$$
g^{\hbar}(\mathbf{v}, \mathbf{y}) \leq \sqrt{m}|\mathbf{y}|^{2}\left(\int_{0}^{1} \sum_{j=1}^{m} \mathbb{E}_{\mathbf{y}, \mathbf{v}}\left[\left|Y_{1,1, j}^{\hbar}(t \mathbf{y})\right|^{4}\right] d t\right)^{\frac{1}{2}} \mathbb{E}_{\mathbf{y}, \mathbf{v}}\left[\left\|\nabla^{2} Y^{\hbar}(0)\right\|^{4(m-1)}\right]^{\frac{1}{2}} .
$$

Step 2 will be completed once we prove the following result.

Lemma A.1. There exist $\hbar_{3}>0, r_{3}>0$ and $C_{3}>0$ such that for any $\hbar \leq h_{3}$ and any $\mathbf{v} \in \mathbb{R}^{m}$ we have

$$
\begin{aligned}
\mathbb{E}_{\mathbf{y}, \mathbf{v}}\left[\left\|\nabla^{2} Y^{\hbar}(0)\right\|^{4(m-1)}\right] & \leq C_{3}(1+|\mathbf{v}|)^{4(m-1)}, \quad|\mathbf{y}|<r_{3}, \\
\mathbb{E}_{\mathbf{y}, \mathbf{v}}\left[\left|Y_{1,1, j}^{\hbar}(t \mathbf{y})\right|^{4}\right] & \leq C_{3}(1+|\mathbf{v}|)^{4}, \quad \forall j,|\mathbf{y}|<r_{3}, t \in[0,1] .
\end{aligned}
$$

Proof. The random matrix $\nabla^{2} Y^{\hbar}$, conditioned by $C_{\mathbf{y}}(\mathbf{v})$, is Gaussian. The same is true of $Y_{1,1, j}^{\hbar}(t \mathbf{y})$ so it suffices to show that there exist $\hbar_{3}>0, r_{3}>0$ and $C_{3}>0$ such that, for any $\hbar \leq h_{3}$, and any $\mathbf{v} \in \mathbb{R}^{m}$ we have

$$
\begin{aligned}
\mathbb{E}_{\mathbf{y}, \mathbf{v}}\left[\left|Y_{i, j}^{\hbar}(0)\right|^{2}\right] & \leq C_{3}(1+|\mathbf{v}|)^{2}, \quad \forall i, j,|\mathbf{y}|<r_{3}, \\
\mathbb{E}_{\mathbf{y}, \mathbf{v}}\left[\left|Y_{1,1, j}^{\hbar}(t \mathbf{y})\right|^{2}\right] & \leq C_{3}(1+|\mathbf{v}|)^{2}, \quad \forall j,|\mathbf{y}|<r_{3}, t \in[0,1] .
\end{aligned}
$$

As in [31], we introduce the index sets

$$
\mathbf{J}=\{ \pm 1, \pm 2, \ldots, \pm m\}, \quad \mathbf{J}_{+}=\mathbf{J} \cap(0, \infty), \quad \mathbf{J}_{-}=\mathbf{J} \cap(-\infty, 0) .
$$

We consider the $\mathbb{R}^{m} \oplus \mathbb{R}^{m}$ valued random Gaussian vector $G^{\hbar}(t)=G_{-}^{\hbar} \oplus G_{+}^{\hbar}, t \in[0,1]$, where

$$
G_{-}^{\hbar}:=\sum_{i=1}^{m} G_{-i}^{\hbar} \mathbf{e}_{i}=\nabla Y^{\hbar}(0), \quad G_{+}^{\hbar}:=\sum_{j=1}^{m} G_{j}^{\hbar} \mathbf{e}_{j}=\nabla Y^{\hbar}(t \mathbf{y}) .
$$

The covariance form of this vector is the $2 m \times 2 m$ symmetric matrix

$$
\begin{aligned}
& S_{\hbar}=S_{\hbar}(t)=\left[\begin{array}{cc}
S_{\hbar}^{-,-} & S_{\hbar}^{-,+} \\
S_{\hbar}^{+,-} & S_{\hbar}^{+,+}
\end{array}\right]=\left[\begin{array}{ll}
A_{\hbar} & B_{\hbar} \\
B_{\hbar} & A_{\hbar}
\end{array}\right], \\
& A_{\hbar}=-\nabla^{2} V^{\hbar}(0), \quad B_{\hbar}=B_{\hbar}(t, \mathbf{y})=-\nabla^{2} V^{\hbar}(t \mathbf{y}) .
\end{aligned}
$$

From (A.3b), we deduce that $S_{\hbar}$ is invertible if $\hbar<\hbar_{1}$ and $|\mathbf{y}| \leq r_{1}$. Its inverse has the block form

$$
\left[\begin{array}{cc}
C_{\hbar}(t) & -D_{\hbar}(t) \\
-D_{\hbar}(t) & C_{\hbar}(t)
\end{array}\right]=\left[\begin{array}{cc}
C_{\hbar}(t) & -A_{\hbar}^{-1} B_{\hbar}(t) C_{\hbar}(t) \\
-A_{\hbar}^{-1} B_{\hbar}(t) C_{\hbar}(t) & C_{\hbar}(t)
\end{array}\right],
$$


where,

$$
C_{\hbar}(t)=C_{\hbar}(t, \mathbf{y}):=\left(A_{\hbar}-B_{\hbar}(t, \mathbf{y}) A_{\hbar}^{-1} B_{\hbar}(t, \mathbf{y})\right)^{-1} .
$$

In [31], Lemma 3.6, we have shown that there exists $\hbar_{3} \in\left(0, \hbar_{1}\right)$ such that the $m \times m$ matrix

$$
K^{\hbar}:=\left(K_{i j}^{\hbar}\right)_{1 \leq i, j \leq m}, \quad K_{i j}^{\hbar}:=V_{1,1, i, j}^{\hbar}(0)
$$

is invertible for $\hbar \in\left[0, \hbar_{2}\right]$ and

$$
\lim _{t \rightarrow 0} t^{2} C_{\hbar}(t, \mathbf{y})=\left(K^{\hbar}\right)^{-1}, \quad \text { uniformly in } \hbar \in\left[0, \hbar_{3}\right] \text { and }|\mathbf{y}| \leq r_{1} .
$$

Next, observe that

$$
\begin{aligned}
C_{\hbar}(t, \mathbf{y})-D_{\hbar}(t, \mathbf{y}) & =A_{\hbar}^{-1}\left(A_{\hbar}-B_{\hbar}(t, \mathbf{y})\right) C_{\hbar}(t, \mathbf{y}) \\
& =A_{\hbar}^{-1} \frac{1}{t^{2}}\left(A_{\hbar}-B_{\hbar}(t, \mathbf{y})\right) t^{2} C_{\hbar}(t, \mathbf{y}),
\end{aligned}
$$

and

$$
\frac{1}{t^{2}}\left(A_{\hbar}-B_{\hbar}(t, \mathbf{y})\right)=\frac{1}{t^{2}}\left(\nabla^{2} V^{\hbar}(t \mathbf{y})-\nabla^{2} V^{\hbar}(0)\right) .
$$

Since the function

$$
\mathbf{x} \mapsto \nabla^{2} V^{\hbar}(\mathbf{x})
$$

is even and $V^{\hbar} \rightarrow V^{0}$ in the $C^{\infty}$-topology as $\hbar \rightarrow 0$ we deduce that the limit

$$
\lim _{t \rightarrow 0} \frac{1}{t^{2}}\left(\nabla^{2} V^{\hbar}(t \mathbf{y})-\nabla^{2} V^{\hbar}(0)\right)
$$

exists, it is finite and it is uniform in $\hbar \in\left[0, \hbar_{3}\right]$ and $|\mathbf{y}| \leq r_{1}$. Using (A.10), we conclude that there exists a constant $c_{1}>0$ such that

$$
\left\|C_{\hbar}(t, \mathbf{y})-D_{\hbar}(t, \mathbf{y})\right\| \leq c_{1}, \quad \forall t \in[0,1], \hbar \in\left[0, \hbar_{3}\right],|\mathbf{y}| \leq r_{1} .
$$

We can now prove (A.9a) and (A.9b).

Proof of (A.9a). Fix $i_{0}, j_{0} \in\{1, \ldots, m\}$. The random variable $Y_{i_{0}, j_{0}}^{\hbar}(0)$, conditioned by $C_{\mathbf{y}}(\mathbf{v})$, is a normal random variable $\bar{Y}_{i_{0}, j_{0}}^{\hbar}$, and its mean and variance are determined by the regression formula, [11], Proposition 1.2. To apply this formula, we need to compute the correlations between $Y_{i_{0}, j_{0}}^{\hbar}(0)$ and $G^{\hbar}$. These are given by the expectations

$$
\boldsymbol{\Xi}_{j}^{\hbar}=\boldsymbol{\Xi}_{j}^{\hbar}(\mathbf{y})=\mathbb{E}\left[Y_{i_{0}, j_{0}}^{\hbar}(0) G_{j}(1)\right], \quad j \in \mathbf{J}
$$

We have

$$
\boldsymbol{\Xi}_{j}^{\hbar}(\mathbf{y})= \begin{cases}V_{i_{0}, j_{0},|j|}^{\hbar}(0), & j \in \mathbf{J}_{-}, \\ V_{i_{0}, j_{0}, j}^{\hbar}(\mathbf{y}), & j \in \mathbf{J}_{+}\end{cases}
$$


We regard the collection $\left(\boldsymbol{\Xi}_{j}^{\hbar}(\mathbf{y})\right)_{j \in \mathbf{J}}$ as a linear map

$$
\mathbf{\Xi}^{\hbar}(\mathbf{y}): \mathbb{R}^{m} \oplus \mathbb{R}^{m} \rightarrow \mathbb{R}, \quad \mathbf{\Xi}^{\hbar}\left(\left(z_{j}\right)_{j \in \mathbf{J}}\right)=\sum_{j \in \mathbf{J}} \boldsymbol{\Xi}_{j}^{\hbar}(\mathbf{y}) z_{j}
$$

In particular, we think of $\boldsymbol{\Xi}^{\hbar}$ as a row vector so its transpose $\left(\boldsymbol{\Xi}^{\hbar}\right)^{\top}$ is a column vector.

Observe that since the function $V^{\hbar}$ is even, the third order derivative $V_{i j k}^{\hbar}$ are odd functions. Thus,

$$
V_{i_{0}, j_{0}, j}^{\hbar}(0)=0
$$

and there exists $r_{3} \in\left(0, r_{1}\right)$ and $c_{3}>0$ such that

$$
\left|V_{i, j, k}^{\hbar}(\mathbf{y})\right| \leq c_{2}|\mathbf{y}|, \quad \forall i, j, k, \forall \hbar \in\left[0, \hbar_{3}\right],|\mathbf{y}| \leq r_{3} .
$$

Hence,

$$
\left\|\mathbf{\Xi}^{\hbar}(\mathbf{y})\right\| \leq c_{3}|\mathbf{y}|, \quad \forall \hbar \in\left[0, \hbar_{3}\right],|\mathbf{y}| \leq r_{3} .
$$

Denote by $\hat{\mathbf{v}} \in \mathbb{R}^{m} \oplus \mathbb{R}^{m}$ the vector $\mathbf{v} \oplus \mathbf{v}$.

According to the regression formula, the mean of the conditioned random variable $\bar{Y}_{i_{0}, j_{0}}^{\hbar}$ is

$$
\mathbb{E}\left[\bar{Y}_{i_{0}, j_{0}}^{\hbar}\right]=-\mathbf{\Xi}^{\hbar}(\mathbf{y})\left(S_{\hbar}^{-1} \hat{\mathbf{v}}\right)=\mathbf{\Xi}^{\hbar}(\mathbf{y})\left(\left(C_{\hbar}-D_{\hbar}\right) \mathbf{v} \oplus\left(C_{\hbar}-D_{\hbar}\right) \mathbf{v}\right)
$$

Using (A.11) and (A.12), we deduce that there exists $c_{3}>0$ such that

$$
\left|\mathbb{E}_{\mathbf{y}, \mathbf{v}}\left[Y_{i_{0}, j_{0}}^{\hbar}\right]\right| \leq c_{3}|\mathbf{y}||\mathbf{v}|, \quad \forall \hbar \in\left[0, \hbar_{3}\right],|\mathbf{y}| \leq r_{3}, \mathbf{v} \in \mathbb{R}^{m}
$$

According to the regression formula, the variance of the conditioned random variable $\bar{Y}_{i_{0}, j_{0}}^{\hbar}$ is

$$
\begin{aligned}
\operatorname{var}\left[\bar{Y}_{i_{0}, j_{0}}^{\hbar}\right] & =\operatorname{var}\left[Y_{i_{0}, j_{0}}^{\hbar}\right]-\boldsymbol{\Xi}^{\hbar}(\mathbf{y}) S_{\hbar}^{-1}\left(\boldsymbol{\Xi}^{\hbar}(\mathbf{y})\right)^{\top} \\
& =V_{i_{0}, j_{0}, i_{0}, j_{0}}^{\hbar}(0)-\boldsymbol{\Xi}^{\hbar}(\mathbf{y}) S_{\hbar}^{-1}\left(\boldsymbol{\Xi}^{\hbar}(\mathbf{y})\right)^{\top} .
\end{aligned}
$$

Using (1.13), (A.11) and (A.12), we deduce that there exists $c_{4}>0$ such that

$$
\operatorname{var}_{\mathbf{y}, \mathbf{v}}\left[Y_{i_{0}, j_{0}}^{\hbar}\right] \leq c_{4}|\mathbf{v}|, \quad \forall \hbar \in\left[0, \hbar_{3}\right],|\mathbf{y}| \leq r_{3}, \mathbf{v} \in \mathbb{R}^{m}
$$

The inequality (A.9a) now follows from (A.13) and (A.14).

Proof of (A.9b). Fix $j_{0} \in\{1,2, \ldots, m\}$. The random variable $Y_{1,1, j_{0}}^{\hbar}(t \mathbf{y})$, conditioned by $C_{\mathbf{y}}(\mathbf{v})$ is a normal random variable $\bar{Y}_{1,1, j_{0}}^{\hbar}$. To describe its mean and its variance, we need to compute the correlations

$$
\boldsymbol{\Omega}_{j}^{\hbar}(t, \mathbf{y}):=\mathbb{E}\left[Y_{1,1, j_{0}}^{\hbar}(t \mathbf{y}) G_{j}^{\hbar}\right]= \begin{cases}-V_{1,1, j_{0},|j|}^{\hbar}(t \mathbf{y}), & j \in \mathbf{J}_{-}, \\ -V_{1,1, j_{0}, j}^{\hbar}((1-t) \mathbf{y}), & j \in \mathbf{J}_{+} .\end{cases}
$$


Again, we think of the collection $\left(\boldsymbol{\Omega}_{j}^{\hbar}(t, \mathbf{y})\right)_{j \in \mathbf{J}}$ as defining a linear map

$$
\boldsymbol{\Omega}^{\hbar}: \mathbb{R}^{m} \oplus \mathbb{R}^{m} \rightarrow \mathbb{R}
$$

The row vector $\boldsymbol{\Omega}^{\hbar}$ splits as a direct sum of row vectors

$$
\boldsymbol{\Omega}^{\hbar}=\boldsymbol{\Omega}_{-}^{\hbar} \oplus \boldsymbol{\Omega}_{+}^{\hbar}, \quad \boldsymbol{\Omega}_{ \pm}^{\hbar}=\left(\boldsymbol{\Omega}_{j}^{\hbar}\right)_{j \in \mathbf{J}_{ \pm}} .
$$

The mean of the random variable $\bar{Y}_{1,1, j_{0}}^{\hbar}$ is

$$
\mathbb{E}\left[\bar{Y}_{1,1, j_{0}}^{\hbar}\right]=-\boldsymbol{\Omega}^{\hbar}\left(\left(C_{\hbar}-D_{\hbar}\right) \mathbf{v} \oplus\left(C_{\hbar}-D_{\hbar}\right) \mathbf{v}\right)
$$

We conclude as before that

$$
\left|\mathbb{E}\left[Y_{1,1, j_{0}}^{\hbar}(t \mathbf{y})\right]\right|=O(|\mathbf{v}| \cdot|\mathbf{y}|), \quad|\mathbf{y}| \leq r_{3}, \mathbf{v} \in \mathbb{R}^{m}, t \in[0,1],
$$

where the constant implied by the $O$-symbol is independent of $\hbar$ and $t$. This convention will stay in place for the remainder of this proof.

Next, we have

$$
\begin{aligned}
\operatorname{var} & \left.\bar{Y}_{1,1, j_{0}}^{\hbar}\right] \\
= & \operatorname{var}\left[Y_{1,1, j_{0}}^{\hbar}(t \mathbf{y})\right]-\boldsymbol{\Omega}^{\hbar} S_{\hbar}^{-1}\left(\boldsymbol{\Omega}^{\hbar}\right)^{\top} \\
= & V_{1,1, j_{0}, 1,1, j_{0}}^{\hbar}(t \mathbf{y})-\left[\boldsymbol{\Omega}_{-}^{\hbar} \boldsymbol{\Omega}_{+}^{\hbar}\right] \cdot\left[\begin{array}{cc}
C_{\hbar} & -D_{\hbar} \\
-D_{\hbar} & C_{\hbar}
\end{array}\right] \cdot\left[\begin{array}{c}
\left(\boldsymbol{\Omega}_{-}^{\hbar}\right)^{\top} \\
\left(\boldsymbol{\Omega}_{+}^{\hbar}\right)^{\top}
\end{array}\right] \\
= & V_{1,1,1,1, j_{0}, j_{0}}^{\hbar}(t \mathbf{y})-\left[\boldsymbol{\Omega}_{-}^{\hbar} \boldsymbol{\Omega}_{+}^{\hbar}\right] \cdot\left[\begin{array}{c}
C_{\hbar}\left(\boldsymbol{\Omega}_{-}^{\hbar}\right)^{\top}-D_{\hbar}\left(\boldsymbol{\Omega}_{+}^{\hbar}\right)^{\top} \\
-D_{\hbar}\left(\boldsymbol{\Omega}_{-}^{\hbar}\right)^{\top}+C_{\hbar}\left(\boldsymbol{\Omega}_{+}^{\hbar}\right)^{\top}
\end{array}\right] \\
= & V_{1,1,1,1, j_{0}, j_{0}}^{\hbar}(t \mathbf{y})-\left(\boldsymbol{\Omega}_{-}^{\hbar} C_{\hbar}\left(\boldsymbol{\Omega}_{-}^{\hbar}\right)^{\top}-\boldsymbol{\Omega}_{-}^{\hbar} D_{\hbar}\left(\boldsymbol{\Omega}_{+}^{\hbar}\right)^{\top}-\boldsymbol{\Omega}_{+}^{\hbar} D_{\hbar}\left(\boldsymbol{\Omega}_{-}^{\hbar}\right)^{\top}+\boldsymbol{\Omega}_{+}^{\hbar} C_{\hbar}\left(\boldsymbol{\Omega}_{+}^{\hbar}\right)^{\top}\right), \\
\left(D_{\hbar}=\right. & \left.C_{\hbar}+O(1)\right) \\
= & V_{1,1,1,1, j_{0}, j_{0}}^{\hbar}(t \mathbf{y})-\left(\boldsymbol{\Omega}_{-}^{\hbar} C_{\hbar}\left(\boldsymbol{\Omega}_{-}^{\hbar}\right)^{\top}-\boldsymbol{\Omega}_{-}^{\hbar} C_{\hbar}\left(\boldsymbol{\Omega}_{+}^{\hbar}\right)^{\top}-\boldsymbol{\Omega}_{+}^{\hbar} C_{\hbar}\left(\boldsymbol{\Omega}_{-}^{\hbar}\right)^{\top}+\boldsymbol{\Omega}_{+}^{\hbar} C_{\hbar}\left(\boldsymbol{\Omega}_{+}^{\hbar}\right)^{\top}\right) \\
& +O(1) \\
= & V_{1,1,1,1, j_{0}, j_{0}}^{\hbar}(t \mathbf{y})-\left(\left(\boldsymbol{\Omega}_{-}^{\hbar}-\boldsymbol{\Omega}_{+}^{\hbar}\right) C_{\hbar}\left(\boldsymbol{\Omega}_{-}^{\hbar}\right)^{\top}-\left(\boldsymbol{\Omega}_{-}^{\hbar}-\boldsymbol{\Omega}_{+}^{\hbar}\right) C_{\hbar}\left(\boldsymbol{\Omega}_{+}^{\hbar}\right)^{\top}\right)+O(1) \\
= & V_{1,1,1,1, j_{0}, j_{0}}^{\hbar}(t \mathbf{y})-\left(\boldsymbol{\Omega}_{-}^{\hbar}-\boldsymbol{\Omega}_{+}^{\hbar}\right) C_{\hbar}\left(\boldsymbol{\Omega}_{-}^{\hbar}-\boldsymbol{\Omega}_{+}^{\hbar}\right)^{\top}+O(1) .
\end{aligned}
$$

Now observe that $\Phi^{\hbar}=\boldsymbol{\Omega}_{-}^{\hbar}-\boldsymbol{\Omega}_{+}^{\hbar}$ is the $m$-dimensional row vector with components

$$
\Phi_{j}^{\hbar}(t, \mathbf{y})=V_{1,1, j_{0}, j}^{\hbar}((1-t) \mathbf{y})-V_{1,1, j_{0}, j}^{\hbar}(t \mathbf{y}),
$$


that satisfy

$$
\left|\Phi_{j}^{\hbar}(t, \mathbf{y})\right|=O(|\mathbf{y}|), \quad \forall j .
$$

From (A.10), we deduce

$$
\left\|C_{\hbar}(t, \mathbf{y})\right\|=O\left(|\mathbf{y}|^{-2}\right)
$$

so that

$$
\left|\left(\boldsymbol{\Omega}_{-}^{\hbar}-\boldsymbol{\Omega}_{+}^{\hbar}\right) C_{\hbar}\left(\boldsymbol{\Omega}_{-}^{\hbar}-\boldsymbol{\Omega}_{+}^{\hbar}\right)^{\top}\right|=O(1) .
$$

This completes the proof of (A.9b) and thus of Lemma A.1 and of statement (i) in Proposition 2.1 .

Step 3. The map

$$
\mathbf{v} \mapsto \mathbb{E}\left[\mathbf{Z}^{\hbar}(\mathbf{v}, B)\left(\mathbf{Z}^{\hbar}(\mathbf{v}, B)-1\right)\right]
$$

is continuous. This follows by using the argument in Point 2 in the proof of [17], Proposition 1.1. Combined with (A.1) will prove the statement (ii) in Proposition 2.1.

Step 4. Prove the statement (iii) in Proposition 2.1. This follows by using the argument in Point 3 in the proof of [17], Proposition 1.1.

Step 5. Using the results in Step 1 and Step 2 and the dominated convergence theorem we obtain the statement (iv).

\section{Notation}

- We set

$$
\mathbb{N}:=\{n \in \mathbb{Z} ; n>0\}, \quad \mathbb{N}_{0}:=\{n \in \mathbb{Z} ; n \geq 0\} .
$$

- $\mathbf{1}_{A}$ denotes the characteristic function of a subset $A$ of a set $S$,

$$
\mathbf{1}_{A}: S \rightarrow\{0,1\}, \quad \mathbf{1}_{A}(a)= \begin{cases}1, & a \in A, \\ 0, & a \in S \backslash A .\end{cases}
$$

- For a topological space $X$, we denote by $\mathcal{B}(X)$ the $\sigma$-algebra of Borel subsets of $X$.

- We will write $N \sim \mathcal{N}(m, v)$ to indicate that $N$ is a normal random variable with mean $m$ and variance $v$.

- For $\mathbf{x}, \mathbf{y} \in \mathbb{R}^{m}$, we set

$$
|\mathbf{x}|_{\infty}:=\max _{1 \leq j \leq m}\left|x_{j}\right|, \quad(\mathbf{x}, \mathbf{y})=\sum_{j=1}^{m} x_{j} y_{j}, \quad|\mathbf{x}|:=\sqrt{(\mathbf{x}, \mathbf{x})} .
$$

- We denote by $\mathbb{A}^{m}$ the affine lattice

$$
\mathbb{A}^{m}=\left(\frac{1}{2}+\mathbb{Z}\right)^{m}
$$


- For any matrix $A$, we denote by $A^{\top}$ its transpose, and by $\|A\|$ its norm

$$
\|A\|=\sup _{|\mathbf{x}|=1}|A \mathbf{x}| .
$$

- We denote by $\mathbb{1}_{m}$ the identity operator $\mathbb{R}^{m} \rightarrow \mathbb{R}^{m}$.

- For any Borel subset $B \subset \mathbb{R}^{m}$ we denote by $|B|$ its Lebesgue measure.

- We denote by $\boldsymbol{\gamma}$ the canonical Gaussian measure on $\mathbb{R}$

$$
\boldsymbol{\gamma}(d x)=\frac{1}{\sqrt{2 \pi}} e^{-\frac{x^{2}}{2}} d x,
$$

and by $\boldsymbol{\Gamma}$ the canonical Gaussian measure on $\mathbb{R}^{m}$

$$
\boldsymbol{\Gamma}(d \mathbf{x})=(2 \pi)^{-\frac{m}{2}} e^{-\frac{|x|^{2}}{2}} d \mathbf{x}
$$

- If $C$ is a symmetric, nonnegative definite $m \times m$ matrix, we write $N \sim \mathcal{N}(0, C)$ to indicate that $N$ is an $\mathbb{R}^{m}$-valued Gaussian random vector with mean 0 and covariance form $C$.

- If $f: \mathbb{R}^{m} \rightarrow \mathbb{R}$ is a twice differentiable function, and $\mathbf{x} \in \mathbb{R}^{m}$, then we denote by $\nabla^{2} f(\mathbf{x})$ its Hessian, viewed as a symmetric operator $\mathbb{R}^{m} \rightarrow \mathbb{R}^{m}$.

\section{Acknowledgments}

I want to thank the anonymous referees for the helpful critique, comments and suggestions.

\section{References}

[1] Adler, R.J. and Naizat, G. (2015). A central limit theorem for the Euler integral of a Gaussian random field. Preprint. Available at arXiv:1506.08772.

[2] Adler, R.J. and Taylor, J.E. (2007). Random Fields and Geometry. Springer Monographs in Mathematics. New York: Springer. MR2319516

[3] Arcones, M.A. (1994). Limit theorems for nonlinear functionals of a stationary Gaussian sequence of vectors. Ann. Probab. 22 2242-2274. MR1331224

[4] Arnold, V.I. (2006). Statistics and classification of topologies of periodic functions and trigonometric polynomials. Proc. Steklov Inst. Math. S13-S23. MR2246984

[5] Arnold, V.I. (2007). Topological classification of trigonometric polynomials of the affine Coxeter group $\tilde{A}_{2}$. Tr. Mat. Inst. Steklova 258 7-16. MR2400519

[6] Arnold, V.I. (2007). Topological classification of Morse functions and generalisations of Hilbert's 16-th problem. Math. Phys. Anal. Geom. 10 227-236. MR2368960

[7] Arnold, V.I. (2015). Experimental Mathematics. MSRI Mathematical Circles Library 16. Providence, RI: Amer. Math. Soc. MR3309308

[8] Azaïs, J.-M., Dalmao, F. and León, J.R. (2016). CLT for the zeros of classical random trigonometric polynomials. Ann. Inst. Henri Poincaré Probab. Stat. 52 804-820. MR3498010

[9] Azaïs, J.-M., Dalmao, F., León, J.R., Nourdin, I. and Poly, G. (2015). Local universality of the number of zeros of random trigonometric polynomials with continuous coefficients. Preprint. Available at arXiv:1512.05583. 
[10] Azaiis, J.-M. and León, J.R. (2013). CLT for crossings of random trigonometric polynomials. Electron. J. Probab. 18 no. 68, 17. MR3084654

[11] Azaïs, J.-M. and Wschebor, M. (2009). Level Sets and Extrema of Random Processes and Fields. Hoboken, NJ: Wiley. MR2478201

[12] Bernstein, D.N. (1975). The number of roots of a system of equations. Funct. Anal. Appl. 9 1-4. MR0435072

[13] Breuer, P. and Major, P. (1983). Central limit theorems for nonlinear functionals of Gaussian fields. J. Multivariate Anal. 13 425-441. MR0716933

[14] Chambers, D. and Slud, E. (1989). Central limit theorems for nonlinear functionals of stationary Gaussian processes. Probab. Theory Related Fields 80 323-346. MR0976529

[15] Cuzick, J. (1976). A central limit theorem for the number of zeros of a stationary Gaussian process. Ann. Probab. 4 547-556. MR0420809

[16] Dalmao, F. (2015). Asymptotic variance and CLT for the number of zeros of Kostlan Shub Smale random polynomials. C. R. Math. Acad. Sci. Paris 353 1141-1145. MR3427922

[17] Estrade, A. and León, J.R. (2015). A central limit theorem for the Euler characteristic of a Gaussian excursion set. Ann. Probab. 44 3849-3878.

[18] Gelfand, I.M. and Vilenkin, N.Ya. (1964 [1977]). Generalized Functions, Vol. 4. New York: Academic Press. MR0435834

[19] Granville, A. and Wigman, I. (2011). The distribution of the zeros of random trigonometric polynomials. Amer. J. Math. 133 295-357. MR2797349

[20] Hörmander, L. (2003). The Analysis of Linear Partial Differential Operators. i. Classics in Mathematics. Berlin: Springer. MR1996773

[21] Horn, R.A. and Johnson, C.R. (1985). Matrix Analysis. Cambridge: Cambridge Univ. Press. MR0832183

[22] Janson, S. (1997). Gaussian Hilbert Spaces. Cambridge Tracts in Mathematics 129. Cambridge: Cambridge Univ. Press. MR1474726

[23] Kouchnirenko, A.G. (1976). Polyèdres de Newton et nombres de Milnor. Invent. Math. 32 1-31. MR0419433

[24] Kratz, M.F. and León, J.R. (1997). Hermite polynomial expansion for non-smooth functionals of stationary Gaussian processes: Crossings and extremes. Stochastic Process. Appl. 66 237-252. MR1440400

[25] Kratz, M.F. and León, J.R. (2001). Central limit theorems for level functionals of stationary Gaussian processes and fields. J. Theoret. Probab. 14 639-672. MR1860517

[26] Major, P. (1981). Multiple Wiener-Itô Integrals. Lecture Notes in Math. 849. Berlin: Springer. MR0611334

[27] Malevich, T.L. (1969). Asymptotic normality of the number of crossings of the zero level by a Gaussian process. Theory Probab. Appl. 14 287-295.

[28] Malliavin, P. (1995). Integration and Probability. Graduate Texts in Mathematics 157. New York: Springer. MR1335234

[29] Meschenmoser, D. and Shashkin, A. (2013). Functional central limit theorem for the measures of level surfaces of the Gaussian random field. Theory Probab. Appl. 57 162-172. MR3201646

[30] Nicolaescu, L.I. (2012). Random Morse functions and spectral geometry. Preprint. Available at arXiv:1209.0639.

[31] Nicolaescu, L.I. (2015). Critical points of multidimensional random Fourier series: Variance estimates. J. Math. Phys. 57 no. 8, 083304, 42 pp.

[32] Nicolaescu, L.I. (2015). A CLT concerning critical points of random functions on a Euclidean space. Stochastic Process. Appl. To appear. Available at DOI:10.1016/j.spa.2017.02.009. 
[33] Nourdin, I. and Peccati, G. (2009). Stein's method on Wiener chaos. Probab. Theory Related Fields 145 75-118. MR2520122

[34] Nourdin, I. and Peccati, G. (2012). Normal Approximations with Malliavin Calculus. from Stein's Method to Universality. Cambridge Tracts in Mathematics 192. Cambridge: Cambridge Univ. Press. MR2962301

[35] Nourdin, I., Peccati, G. and Podolskij, M. (2011). Quantitative Breuer-Major theorems. Stochastic Process. Appl. 121 793-812. MR2770907

[36] Nualart, D. and Peccati, G. (2005). Central limit theorems for sequences of multiple stochastic integrals. Ann. Probab. 33 177-193. MR2118863

[37] Peccati, G. and Tudor, C.A. (2005). Gaussian limits for vector-valued multiple stochastic integrals. In Séminaire de Probabilités XXXVIII. Lecture Notes in Math. 1857 247-262. Berlin: Springer. MR2126978

[38] Slud, E. (1991). Multiple Wiener-Itô integral expansions for level-crossing-count functionals. Probab. Theory Related Fields 87 349-364. MR1084335

[39] Slud, E.V. (1994). MWI representation of the number of curve-crossings by a differentiable Gaussian process, with applications. Ann. Probab. 22 1355-1380. MR1303648

[40] Sodin, M. and Tsirelson, B. (2004). Random complex zeroes. I. Asymptotic normality. Israel J. Math. 144 125-149. MR2121537

Received February 2016 and revised May 2016 